\title{
Geometry of Configuration Spaces of Tensegrities
}

\author{
Franck Doray • Oleg Karpenkov • Jan Schepers
}

Received: 20 June 2008 / Revised: 26 February 2009 / Accepted: 23 July 2009 /

Published online: 18 November 2009

(C) Springer Science+Business Media, LLC 2009

\begin{abstract}
Consider a graph $G$ with $n$ vertices. In this paper we study geometric conditions for an $n$-tuple of points in $\mathbb{R}^{d}$ to admit a nonzero self-stress with underlying graph $G$. We introduce and investigate a natural stratification, depending on $G$, of the configuration space of all $n$-tuples in $\mathbb{R}^{d}$. In particular we find surgeries on graphs that give relations between different strata. Further we discuss questions related to geometric conditions defining the strata for plane tensegrities. We conclude the paper with particular examples of strata for tensegrities in the plane with a small number of vertices.
\end{abstract}

Keywords Tensegrity $\cdot$ Self-stress $\cdot$ Frameworks $\cdot$ Stratification

\section{Introduction}

In his paper [15] J.C. Maxwell made one of the first approaches to the study of equilibrium states for frames under the action of static forces. He noted that the frames together with the forces give rise to reciprocal figures. In the second half of the twentieth century the artist K. Snelson built many surprising sculptures consisting of cables and bars that are actually such frames in equilibrium, see [21]. R. Buckminster Fuller

F. Doray and J. Schepers supported by VICI-grant 639.033.402 of NWO.

O. Karpenkov partially supported by RFBR grant SS-709.2008.1 and by NWO-DIAMANT grant 613.009.001.

F. Doray $(\bowtie) \cdot$ O. Karpenkov · J. Schepers

Mathematisch Instituut, Universiteit Leiden, P.O. Box 9512, 2300 RA Leiden, The Netherlands e-mail: doray@math.leidenuniv.nl

O. Karpenkov

e-mail:karpenk@mccme.ru

J. Schepers

e-mail: janschepers1@gmail.com 
introduced the name "tensegrity" for these constructions, combining the words "tension" and "integrity." A nice overview of the history of tensegrity constructions is made by R. Motro in his book [16].

In mathematics, tensegrities were investigated in several papers. Roth and Whiteley [18] and Connelly and Whiteley [7] studied rigidity and flexibility of tensegrities, see also the survey about rigidity in [26].

White and Whiteley [25] started the investigation of geometric realizability conditions for a tensegrity with prescribed bars and cables. In the preprint [8] M. de Guzmán describes other examples of geometric conditions for tensegrities.

Tensegrities have a wide range of applications in different branches of science and in architecture. For instance, they are used in the study of viruses [5], cells [12], for construction of deployable mechanisms [20, 24], etc.

There are two main ways of working with tensegrities. The first deals with questions on rigidity of tensegrities, and the second with questions on the space of selfstresses on the edges of tensegrities. There is a certain duality between self-stresses and infinitesimal rigidity; more detailed explanations are given in Sect. 2.2. Nevertheless, there are many questions that are natural in the study of self-stresses and do not have nice reformulations in rigidity and vice versa. In this paper we investigate questions about self-stresses. Still, we mention general links to rigidity.

Consider a frame $F$ and the graph $G$ corresponding to the frame $F$. We say that the graph $G$ is realizable as a tensegrity on $F$ if there exists an assignment of nonzero stresses to the edges of the frame such that the frame is in a static equilibrium.

Suppose a graph $G$ is given. Is the graph $G$ realizable as a tensegrity for a frame representing $G$ in general/special position? How many independent realizations does it have? We develop a new technique to study such questions. We introduce special operations (surgeries) that change the graph in a certain way but preserve realizability properties. These surgeries are much in the spirit of matroid theory.

Let $n$ be the number of vertices of $G$. Consider the configuration space of all $n$-tuples of points in $\mathbb{R}^{d}$. We define a natural stratification of the configuration space such that each stratum corresponds to a certain set of admissible tensegrities associated to $G$. Suppose that one wants to obtain a construction with some edges of $G$ replaced by struts and the others by cables, then he/she should take a configuration in a specific stratum of the stratification.

In this paper we prove that all the strata are semialgebraic sets, and therefore a notion of dimension is well defined for them. This allows us to ask the second question above more formally: what is the minimal codimension of the strata in the configuration space that contains $n$-tuples of points admitting a tensegrity with underlying graph $G$ ? Our technique of surgeries on graphs gives the first answers in this case. In particular we obtain the list of all 6,7, and 8 vertex tensegrities in the plane that are realizable for codimension 1 strata. We note that the complete answers to the above questions are not known to the authors.

White and Whiteley [25] and de Guzmán and Orden [9, 10] have found the geometric conditions of realizability of plane tensegrities with 6 vertices and of some other particular cases. We continue the investigation for other graphs (see Sect. 6.2). In all the observed examples the strata are defined by certain systems of geometric conditions. It turns out that all these geometric conditions are obtained from elementary ones: 
- two points coincide;

- three points are on a line;

- five points $a, b, c, d, e$ satisfy: $e$ is the intersection point of the lines passing through points $a$ and $b$, and points $c$ and $d$, respectively. As W. Whiteley pointed out to us, these conditions are sufficient to describe any stratum for planar tensegrities (see Sect. 5).

This paper is organized as follows. We start in Sect. 2 with general definitions. In Sect. 2.1 we describe the configuration space of tensegrities associated to a given graph as a fibration over the affine space of all frameworks. Further in Sect. 2.2 we discuss a duality between spaces of self-stresses and spaces of infinitesimal motions via the rigidity matrix. We introduce a natural stratification on the space of all frameworks and prove that all strata are semialgebraic sets (hence the strata have welldefined dimensions) in Sect. 2.3. In Sect. 3 we study the dimension of solutions for graphs on general configurations of points in $\mathbb{R}^{d}$. Later in this section we calculate the dimensions in the simplest cases and formulate general open questions. In Sect. 4 we study surgeries on graphs and frameworks that induce isomorphisms of the spaces of self-stresses for the frameworks. We give general definitions related to systems of geometric conditions for plane tensegrities in Sect. 5. Any stratum is a dense subset of the solution of one of such systems. Finally in Sect. 6 we give particular examples of graphs and their strata for tensegrities in the plane. We study the dimension of the space of self-stresses in Sect. 6.1 and give tables of geometric conditions for codimension 1 strata for graphs with 8 vertices and less in Sect. 6.2.

\section{General Definitions}

\subsection{Configuration Spaces of Tensegrities}

Recall standard definitions of tensegrities. We take them mostly from [6]. See also [19] for a collection of open problems and a good bibliography. We define $G(P)$ without additional "cable-bar-strut" structure specification (this is more convenient for the aims of the present paper).

Definition 2.1 Fix a positive integer $d$. Let $G=(V, E)$ be an arbitrary graph without loops and multiple edges. Let it have $n$ vertices:

- A configuration is a finite collection $P$ of $n$ labeled points $\left(p_{1}, p_{2}, \ldots, p_{n}\right)$, where each point $p_{i}$ (also called a vertex) is in a fixed Euclidean space $\mathbb{R}^{d}$.

- The pair $G$ and $P$ is called a tensegrity framework and is denoted as $G(P)$.

- We say that a load or force $F$ acting on a framework $G(P)$ in $\mathbb{R}^{d}$ is an assignment of a vector $f_{i}$ in $\mathbb{R}^{d}$ to each vertex $i$ of $G$.

- We say that a stress $w$ for a framework $G(P)$ in $\mathbb{R}^{d}$ is an assignment of a real number $w_{i, j}=w_{j, i}$ (we call it an edge-stress) to each edge $p_{i} p_{j}$ of $G$. An edgestress is regarded as a tension or a compression in the edge $p_{i} p_{j}$. For simplicity reasons, we put $w_{i, j}=0$ if there is no edge between the corresponding vertices. 
We say that $w$ resolves a load $F$ if the following vector equation holds for each vertex $i$ of $G$ :

$$
f_{i}+\sum_{\{j \mid j \neq i\}} w_{i, j}\left(p_{j}-p_{i}\right)=0 .
$$

By $p_{j}-p_{i}$ we denote the vector from the point $p_{i}$ to the point $p_{j}$.

- A stress $w$ is called a self-stress if the following equilibrium condition is fulfilled at every vertex $p_{i}$ :

$$
\sum_{\{j \mid j \neq i\}} w_{i, j}\left(p_{j}-p_{i}\right)=0 .
$$

Actually, this means that the stress resolves the zero force.

- A couple $(G(P), w)$ is called a tensegrity if $w$ is a self-stress for the framework $G(P)$.

Denote by $W(n)$ the linear space of dimension $n^{2}$ of all edge-stresses $w_{i, j}$. Consider a framework $G(P)$ and denote by $W(G, P)$ the subset of $W(n)$ of all possible self-stresses for $G(P)$. By definition of self-stress, the set $W(G, P)$ is a linear subspace of $W(n)$.

Definition 2.2 The configuration space of tensegrities corresponding to the graph $G$ is the set

$$
\Omega_{d}(G):=\left\{(G(P), w) \mid P \in\left(\mathbb{R}^{d}\right)^{n}, w \in W(G, P)\right\} .
$$

The set $\left\{G(P) \mid P \in\left(\mathbb{R}^{d}\right)^{n}\right\}$ is said to be the base of the configuration space; we denote it by $B_{d}(G)$.

If we forget about the edges between the points in all the frameworks, then we get natural bijections between $\Omega_{d}(G)$ and a subset of $\left(\mathbb{R}^{d}\right)^{n} \times W(n)$, and between $B_{d}(G)$ and $\left(\mathbb{R}^{d}\right)^{n}$. Later on we actually identify the last two pairs of sets. The bijections induce natural topologies on $\Omega_{d}(G)$ and $B_{d}(G)$.

Let $\pi$ be the natural projection of $\Omega_{d}(G)$ to the base $B_{d}(G)$. This defines the structure of a fibration. For a given framework $G(P)$ of the base, we call the set $W(G, P)$ the linear fiber at the point $P$ (or at the framework $G(P)$ ) of the configuration space.

Consider a self-stress $w$ for the framework $G(P)$. We say that the edge $p_{i} p_{j}$ is a cable if $w_{i, j}<0$ and a strut if $w_{i, j}>0$. If we do not care about the sign of the edge-stress, we say that this edge is a bar.

Denote by "sgn" the sign function over $\mathbb{R}$.

Definition 2.3 Consider a framework $G(P)$ and one of its self-stresses $w$. The $n \times n$ matrix $\left(\operatorname{sgn}\left(w_{i, j}\right)\right)$ is called the strut-cable matrix of the stress $w$ and denoted by $\operatorname{sgn}(w)$.

Let us give one example of a strut-cable matrix.

Example 2.4 Consider a configuration of four points in the plane: $p_{1}(0,0), p_{2}(1,0)$, $p_{3}(2,2), p_{4}(0,1)$ and a self-stress $w$ as on the picture: $w_{1,2}=6, w_{1,3}=-3$, 
$w_{1,4}=6, w_{2,3}=2, w_{2,4}=-4, w_{3,4}=2$. Then we have

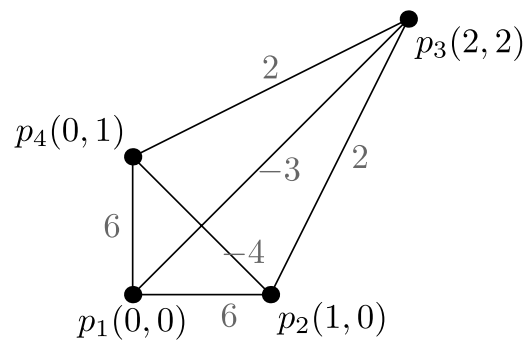

$$
\operatorname{sgn}(w)=\left(\begin{array}{cccc}
0 & 1 & -1 & 1 \\
1 & 0 & 1 & -1 \\
-1 & 1 & 0 & 1 \\
1 & -1 & 1 & 0
\end{array}\right)
$$

\subsection{Rigidity Matrix and Infinitesimal Rigidity}

To study the dimension of the fibers $W(G, P)$ we use the rigidity matrix. Let us briefly recall its definition from [26]. Let $G(P)$ be a framework with vertices $V=$ $\left\{p_{1}, \ldots, p_{n}\right\}$ and edge set $E$. The rigidity matrix $M(G(P))$ of a framework $G(P)$ in dimension $d$ is the $|E| \times d n$ matrix

\begin{tabular}{|c|c|c|c|}
\hline & vertex $a$ & vertex $b$ & vertex $f$ \\
\hline edge $\{a, b\}$ & {$\left[a_{1}-b_{1}, \ldots, a_{d}-b_{d}\right.$} & $b_{1}-a_{1}, \ldots, b_{d}-a_{d}$ & $0, \ldots, 0$ \\
\hline edge $\{a, f\}$ & $a_{1}-f_{1}, \ldots, a_{d}-f_{d}$ & $0, \ldots, 0$ & $\ldots \quad f_{1}-a_{1}, \ldots, f_{d}-a_{d}$ \\
\hline edge $\{e, f\}$ & $0, \ldots, 0$ & $0, \ldots, 0$ & $f_{1}-e_{1}, \ldots, f_{d}-e_{d}$ \\
\hline
\end{tabular}

So, for the edge $\{e, f\}$, the matrix has the row with $e_{1}-f_{1}, \ldots, e_{d}-f_{d}$ in the columns of $e$ and with $f_{1}-e_{1}, \ldots, f_{d}-e_{d}$ in the columns of $f$.

Let us replace all the edges of $G(P)$ by rigid bars with hinges at the vertices. The rigidity matrix gives information on the infinitesimal motions of such a configuration: an infinitesimal motion is defined as a solution of $M(G(P)) X=0$, where we regard $X$ as consisting of $n$ vectors in $\mathbb{R}^{d}$ (one vector at each vertex). Denote the space of all infinitesimal motions of $G(P)$ by $R(G, P)$. Then we have

$$
\operatorname{dim} R(G, P)=d n-\operatorname{rank} M(G(P)) .
$$

We assume now that the affine space spanned by the vertices of the framework is the whole space $\mathbb{R}^{d}$. If $\operatorname{dim} R(G, P)=\frac{d(d+1)}{2}$, then there are no infinitesimal motions other than Euclidean isometries. In that case one calls the framework infinitesimally rigid.

It is interesting to note that the matrix transpose to the rigidity matrix plays a similar role for studying self-stresses:

$$
\operatorname{dim} W(G, P)=|E|-\operatorname{rank}(M(G(P)))^{\mathrm{t}} .
$$

So we can write

$$
\operatorname{dim} W(G, P)-\operatorname{dim} R(G, P)=|E|-d n .
$$

The most interesting case here is where $G(P)$ is infinitesimally rigid and where $|E|$ is minimal (i.e., equal to $n d-\frac{d(d+1)}{2}$ ). In the planary case such frameworks were studied by G. Laman. 
Theorem 2.5 (Laman [14]) Let $G=(V, E)$ be a graph satisfying $2|V|-|E|=3$. Then a framework $G(P)$ on a generic point configuration $P$ is infinitesimally rigid if and only if for every $X \subset V$ with $|X| \geq 2$, the subgraph induced by $X$ has at most $2|X|-3$ edges.

A graph as in this theorem is called an isostatic graph in $\mathbb{R}^{2}$ or a Laman graph. We remark here that in this context one calls a point configuration generic if there is no algebraic relation over the rational numbers between the coordinates of the points. One of the goals of the present paper is to give a more precise meaning to the word "generic" (see Remark 2.8). Laman's theorem immediately implies the following.

Corollary 2.6 Let $G=(V, E)$ be a graph satisfying $2|V|-|E|=3$. Then a framework $G(P)$ on a generic point configuration $P$ has a nonzero self-stress if and only if $G$ is not isostatic in $\mathbb{R}^{2}$.

For additional information about the connection between infinitesimal rigidity and the existence of nonzero self-stresses, we refer to the papers [18] by B. Roth and W. Whiteley and [7] by R. Connelly and W. Whiteley.

\subsection{Stratification of the Base of a Configuration Space of Tensegrities}

Suppose that we have some framework $G(P)$ and we want to find a cable-strut construction on it. Then which edges can be replaced by cables, and which by struts? What is the geometric position of points for which given edges may be replaced by cables and the others by struts? These questions lead to the following definition.

Definition 2.7 A linear fiber $W\left(G, P_{1}\right)$ is said to be equivalent to a linear fiber $W\left(G, P_{2}\right)$ if there exists a homeomorphism $\xi$ between $W\left(G, P_{1}\right)$ and $W\left(G, P_{2}\right)$ such that for any self-stress $w$ in $W\left(G, P_{1}\right)$, the self-stress $\xi(w)$ satisfies

$$
\operatorname{sgn}(\xi(w))=\operatorname{sgn}(w) .
$$

The described equivalence relation gives us a stratification of the base $B_{d}(G)=$ $\left(\mathbb{R}^{d}\right)^{n}$. A stratum is by definition a maximal connected set of points with equivalent linear fibers. Once we have proven Theorem 2.10 , by general theory of semialgebraic sets (see, for instance, [3]) it follows that all strata are path-connected.

Remark 2.8 Using this definition, we can replace "generic point configuration" in Laman's theorem (Theorem 2.5 and Corollary 2.6) by "point configuration in a stratum of codimension 0 in $B_{2}(G)$."

Example 2.9 We describe the stratification of $B_{1}\left(K_{3}\right)=\mathbb{R}^{3}$ for the complete graph $K_{3}$ on three vertices, i.e., the case of 3 -vertex tensegrities on a line. The point $\left(x_{1}, x_{2}, x_{3}\right)$ in $\mathbb{R}^{3}$ corresponds to the framework with vertices $p_{1}=\left(x_{1}\right), p_{2}=\left(x_{2}\right)$, and $p_{3}=\left(x_{3}\right)$.

The stratification consists of 13 strata. There is 1 one-dimensional stratum, and there are 6 two-dimensional and 6 three-dimensional strata. The union of the strata 
Fig. 1 The section $x_{1}=0$ of the stratification of $B_{1}\left(K_{3}\right)$

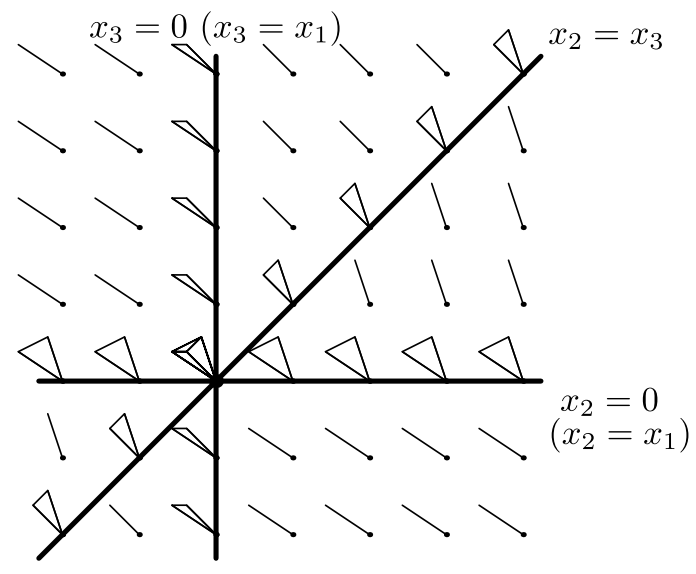

of codimension greater than 1 is the union of three planes meeting in one line, the angles between these planes are all equal to $\pi / 3$. On Fig. 1 we show the plane section $x_{1}=0$ of the stratification. We indicate dimensions of corresponding fibers by segments, triangles, and a tetrahedron in the one-, two-, and three-dimensional cases, respectively.

The one-dimensional stratum consists of frameworks with all vertices coinciding: $x_{1}=x_{2}=x_{3}$. The dimension of the fiber at a point of this stratum is three.

Any of the two-dimensional strata consists of frameworks with exactly two vertices coinciding. The strata are the connected components of the complement to the line $x_{1}=x_{2}=x_{3}$ in the union of the three planes $x_{1}=x_{2}, x_{1}=x_{3}$, and $x_{2}=x_{3}$. The dimension of the fiber at a point of any of these strata is two.

Any of the three-dimensional strata consists of frameworks with distinct vertices. The strata are the connected components of the complement in $\mathbb{R}^{3}$ to the union of the three planes $x_{1}=x_{2}, x_{1}=x_{3}$, and $x_{2}=x_{3}$. The dimension of the fiber at a point of any of these strata is one.

In general we have the following theorem.

Theorem 2.10 Any stratum is a semialgebraic set.

For the definition and basic properties of semialgebraic sets, we refer the reader to [3].

We need two preliminary lemmas for the proof of the theorem, but first we introduce the following notation.

Let $M$ be an arbitrary symmetric $(n \times n)$-matrix with zeroes on the diagonal and all the other entries belonging to $\{-1,0,1\}$. Let $i$ be an integer with $0 \leq i \leq n^{2}$. We say that a couple $(M, i)$ is a stratum symbol.

For an arbitrary framework $G(P)$, we denote by $W_{M}(G, P)$ the set of all selfstresses with strut-cable matrix $\operatorname{sgn}(w)$ equal to $M$. The closure of $W_{M}(G, P)$ is a pointed polyhedral cone with vertex at the origin. The set $W_{M}(G, P)$ is homeomorphic to an open $k$-dimensional disc; we call $k$ the dimension of $W_{M}(G, P)$ and denote it by $\operatorname{dim}\left(W_{M}(G, P)\right)$. 
For any stratum symbol $(M, i)$, we denote by $\Xi(G, M, i)$ the set

$$
\left\{(G(P), w) \mid w \in W(G, P), \operatorname{sgn}(w)=M, \operatorname{dim}\left(W_{M}(G, P)\right)=i\right\} \subset \Omega_{d}(G) .
$$

Lemma 2.11 For any stratum symbol $(M, i)$, the subset $\pi(\Xi(G, M, i))$ of the base $B_{d}(G)$ is either empty or is a semialgebraic set.

Proof The set $\Xi(G, M, i)$ is a semialgebraic set since it is defined by a system of equations and inequalities in the coordinates of the vertices and the edge-stresses of the following three types:

(a) quadratic equilibrium condition equations;

(b) linear equations or inequalities specifying if the coordinate values $w_{i, j}$ are zeroes, positive, or negative reals;

(c) algebraic equations and inequalities defining respectively $\operatorname{dim}\left(W_{M}(G, P)\right) \leq i$ and $\operatorname{dim}\left(W_{M}(G, P)\right) \geq i$. Note that $\operatorname{dim}\left(W_{M}(G, P)\right)$ is equal to the dimension of the linear space spanned by $W_{M}(G, P)$.

Let us make a small remark about item (c). At each framework we take the system of equilibrium conditions and equations of type $w_{i, j}=0$ in the variables $w_{i, j}$. This system consists of the equalities of items (a) and (b). It is linear in the variables $w_{i, j}$. The corresponding matrix here is the rigidity matrix, and its coefficients depend linearly on the coordinates of the framework vertices. The equations and inequalities of item (c) are defined by some determinants of submatrices of the rigidity matrix being equal or not equal to zero. Therefore, they are algebraic.

Since by the Tarski-Seidenberg theorem any projection of a semialgebraic set is semialgebraic, the set $\pi(\Xi(G, M, i))$ is semialgebraic.

Denote by $S(G, P)$ the set of all stratum symbols $(M, i)$ that are realized by the point $G(P)$; in other words,

$$
S(G, P)=\{(M, i) \mid G(P) \in \pi(\Xi(G, M, i))\} .
$$

Lemma 2.12 Let $G\left(P_{1}\right)$ and $G\left(P_{2}\right)$ be two frameworks. Then $S\left(G, P_{1}\right)=S\left(G, P_{2}\right)$ if and only if the linear fiber $W\left(G, P_{1}\right)$ is equivalent to the linear fiber $W\left(G, P_{2}\right)$.

Proof Let the linear fiber at the point $G\left(P_{1}\right)$ be equivalent to the linear fiber at the point $G\left(P_{2}\right)$; then by definition we have

$$
S\left(G, P_{1}\right)=S\left(G, P_{2}\right)
$$

Suppose now that $S\left(G, P_{1}\right)=S\left(G, P_{2}\right)$. Let us denote by $\overline{W\left(G, P_{i}\right)}$ the one-point compactification of the fiber $W\left(G, P_{i}\right)$ for $i=1,2$. So $\overline{W\left(G, P_{i}\right)}$ is homeomorphic to a sphere of dimension $\operatorname{dim} W\left(G, P_{i}\right)$.

For any point $P$ and any $M$, the set $W_{M}(G, P)$ is a convex cone homeomorphic to an open disc of dimension $\operatorname{dim}\left(W_{M}(G, P)\right)$. So, for any point $P$, we have a natural CW-decomposition of $\overline{W(G, P)}$ with cells $W_{M}(G, P)$ and the new one-point cell. 
A cell $W_{M^{\prime}}\left(G, P_{1}\right)$ is adjacent to a cell $W_{M^{\prime \prime}}\left(G, P_{1}\right)$ iff the cell $W_{M^{\prime}}\left(G, P_{2}\right)$ is adjacent to the cell $W_{M^{\prime \prime}}\left(G, P_{2}\right)$. This is true, since the couples of cells corresponding to $M^{\prime}$ and to $M^{\prime \prime}$ are defined by the same sets of equations and inequalities of type $>$, and the closures of $W_{M^{\prime}}\left(G, P_{i}\right)$ for $i=1,2$ are defined by the system defining $W_{M^{\prime}}\left(G, P_{i}\right)$ with all $>$ in the inequalities replaced by $\geq$.

Therefore, there exists a homeomorphism of $\overline{W\left(G, P_{1}\right)}$ and $\overline{W\left(G, P_{2}\right)}$, sending all the cells $W_{M}\left(G, P_{1}\right)$ to the corresponding cells $W_{M}\left(G, P_{2}\right)$. We leave the proof of this statement as an exercise for the reader; this can be done by inductively constructing the homeomorphism on the $k$-skeletons of the CW-complexes.

Hence, the linear fibers $W\left(G, P_{1}\right)$ and $W\left(G, P_{2}\right)$ are equivalent.

Proof of Theorem 2.10 Let us prove the theorem for a stratum containing some point $P$. Consider any point $P^{\prime}$ in the stratum. By definition, $W(G, P)$ is equivalent to the space $W\left(G, P^{\prime}\right)$, and hence by Lemma 2.12, we have $S(G, P)=S\left(G, P^{\prime}\right)$.

Consider the set

$$
\bigcap_{(M, i) \in S(G, P)} \pi(\Xi(G, M, i)) \backslash\left(\bigcup_{(M, i) \notin S(G, P)} \pi(\Xi(G, M, i))\right),
$$

we denote it $\Sigma(P)$. So $\Sigma(P)$ is the set of frameworks $G\left(P^{\prime}\right)$ for which $S\left(G, P^{\prime}\right)=$ $S(G, P)$. By Lemma 2.11 all the sets $\pi(\Xi(G, M, i))$ are semialgebraic. Therefore, the set $\Sigma(P)$ is semialgebraic. Denote by $\Sigma^{\prime}(P)$ the connected component of $\Sigma(P)$ that contains the point $P$. Since the set $\Sigma(P)$ is semialgebraic, the set $\Sigma^{\prime}(P)$ is also semialgebraic, see [3].

Let us show that $\Sigma^{\prime}(P)$ is the stratum of $B_{d}(G)$ containing the point $P$. First, the set $\Sigma^{\prime}(P)$ is contained in the stratum. This holds since $\Sigma^{\prime}(P)$ is connected and consists of points with equivalent sets $S(G, P)$. Hence by Lemma 2.12 all the points of $\Sigma^{\prime}(P)$ have equivalent linear fibers $W(G, P)$. Secondly, the stratum is contained in the space $\Sigma^{\prime}(P)$. This holds since the stratum is connected and consists of points with equivalent linear fibers $W(G, P)$. Thus by Lemma 2.12 all the points of the stratum have equivalent sets $S(G, P)$.

As we have shown, the stratum containing $P$ coincides with $\Sigma^{\prime}(P)$ and hence is semialgebraic.

From the above proof it follows that the total number of strata is finite.

\section{On the Tensegrity $d$-Characteristic of Graphs}

In this section we study the dimension of the linear fiber for graphs on a general point configuration in $\mathbb{R}^{d}$. We give a natural definition of the tensegrity $d$-characteristic of a graph and calculate it for the simplest graphs. We also mention some questions related to connections with rigidity matroids. In addition we formulate general open questions for further investigation. 


\subsection{Definition and Basic Properties of the Tensegrity $d$-Characteristic}

Note that for any two points $P_{1}$ and $P_{2}$ of the same stratum $S$ of the space $B_{d}(G)$ for a graph $G$, we have

$$
\operatorname{dim}\left(W\left(G, P_{1}\right)\right)=\operatorname{dim}\left(W\left(G, P_{2}\right)\right)
$$

Denote this number by $\operatorname{dim}(G, S)$. Denote also by $\operatorname{codim}(S)$ the integer

$$
\operatorname{dim}\left(B_{d}(G)\right)-\operatorname{dim}(S) .
$$

Consider a graph $G$ with at least one edge. We call the integer

$$
\min \left\{\operatorname{codim} S \mid S \text { is a stratum of } B_{d}(G), \operatorname{dim}(G, S)>0\right\}
$$

the codimension of $G$ and denote it by $\operatorname{codim}_{d}(G)$.

Definition 3.1 We call the integer

$\left\{\begin{array}{l}1-\operatorname{codim}_{d}(G) \quad \text { if } \operatorname{codim}_{d}(G)>0, \\ \max \{\operatorname{dim}(W(G, P)) \mid G(P) \text { contained in a codimension zero stratum }\} \text { otherwise }\end{array}\right.$ the tensegrity $d$-characteristic of the graph $G$ (or the $d-T C$ of $G$ for short) and denote it by $\tau_{d}(G)$.

Example 3.2 Consider the two graphs shown on Fig. 2. The left one is a graph of codimension 1 in the plane, and it can be realized as a tensegrity iff either the two triangles are in perspective position or the points of one of the two triples $\left(v_{1}, v_{4}, v_{5}\right)$ or $\left(v_{2}, v_{3}, v_{6}\right)$ lie on a line (for more details, see [10]), so its 2-TC is zero. The graph on the right has a two-dimensional space of self-stresses for a general position plane framework, and hence its 2-TC equals two (we show this later in PropositionExample 6.1). Notice that Corollary 2.6 applies as well to the left graph of this example.

Proposition 3.3 Let $S_{1}$ and $S_{2}$ be two strata of codimension 0 . Let $G\left(P_{1}\right)$ and $G\left(P_{2}\right)$ be two points of the strata $S_{1}$ and $S_{2}$, respectively. Then

$$
\operatorname{dim}\left(W\left(G, P_{1}\right)\right)=\operatorname{dim}\left(W\left(G, P_{2}\right)\right) .
$$
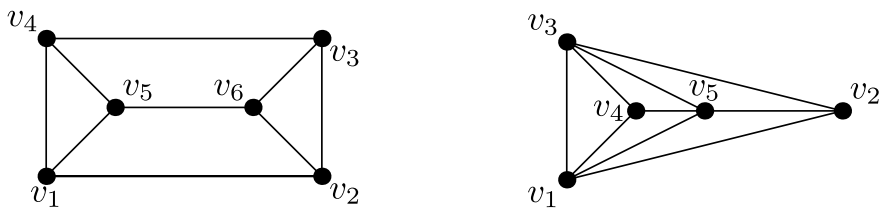

Fig. 2 A graph with zero tensegrity 2-characteristic (the left one) and a graph whose 2-characteristic equals 2 (the right one) 
Proof The equilibrium conditions give a linear system of equations in the variables $w_{i, j}$, at each framework linearly depending on the coordinates of the vertices. The dimension of the solution space is determined by the rank of the matrix of this system. The subset of $B_{d}(G)$ where the rank is not maximal is an algebraic subset of positive codimension. By definition, this set does not have elements in the strata $S_{1}$ and $S_{2}$. This yields the statement of the proposition.

Corollary 3.4 Let $G$ be a graph. If $\tau_{d}(G) \geq 0$, then for every framework $G(P)$ in a codimension 0 stratum, we have $\operatorname{dim} W(G, P)=\tau_{d}(G)$.

\subsection{Atoms and Atom Decomposition}

In this subsection we recall a definition and some results of de Guzmán and Orden [10] that we use later.

Consider a point configuration $P$ of $d+2$ points in general position in $\mathbb{R}^{d}$. Throughout this subsection "general position" means that no $d+1$ of them are contained in a hyperplane. An atom in $\mathbb{R}^{d}$ is a tensegrity $\left(K_{d+2}(P), w\right)$, where $K_{d+2}$ is the complete graph on $d+2$ vertices and where $w$ is a nonzero self-stress.

According to [10, Sect. 2], the linear fiber $W\left(K_{d+2}, P\right)$ is one-dimensional for $P$ in general position; in particular this implies $\tau_{d}\left(K_{d+2}\right)=1$. In addition the edgestress on every edge in the atom is nonzero. A more general statement holds.

Lemma 3.5 [10, Lemma 2.2] Let $G(P)$ be a framework on a point configuration $P$ in $\mathbb{R}^{d}$ in general position. Let $p \in P$. Given a nonzero self-stress on $G(P)$, then either at least $d+1$ of the edges incident to $p$ receive nonzero edge-stress, or all of them have zero edge-stresses.

M. de Guzmán and D. Orden showed that one can consider atoms as the building blocks of tensegrity structures. First, we explain how to add tensegrities. Let $T=$ $(G(P), w)$ and $T^{\prime}=\left(G^{\prime}\left(P^{\prime}\right), w^{\prime}\right)$ be two tensegrities. We define $T+T^{\prime}$ as follows. The framework of $T+T^{\prime}$ is $G(P) \cup G^{\prime}\left(P^{\prime}\right)$, where we take the union of vertices and edges. The edge-stress on a common edge $p_{i} p_{j}=p_{k}^{\prime} p_{l}^{\prime}$ is defined as $w_{i, j}+w_{k, l}^{\prime}$, and on an edge appearing exactly in one of the original frameworks we put the original edge-stress. It is easy to see that the defined stress is a self-stress, so $T+T^{\prime}$ is a tensegrity.

Theorem 3.6 [10, Theorem 3.2] Every tensegrity $(G(P), w)$ with a general position point configuration $P$ and $w_{i, j} \neq 0$ on all edges of $G$ is a finite sum of atoms. This decomposition is not unique in general.

\subsection{Rigidity Matroids, Connelly's Conjecture, and Atom Decomposition}

Let us say a few words about a statement that is a rigidity analogue of atom decomposition for plane tensegrities. For this, we need several definitions.

The rigidity matroid was introduced for studying rigidity questions, and hence it is sometimes related to verifying the existence of nonzero self-stresses, i.e., to determine whether $\tau_{n}>0$ or $\leq 0$ (see Sect. 2.2 and the references mentioned there). 
We restrict ourselves to the geometric description of rigidity matroids in the planar case; the notation and definitions are taken mostly from [13].

For a graph $G=(V, E)$ and a subset $X$ of the vertex set $V$, we denote by $i_{G}(X)$ the number of edges in the subgraph of $G$ induced by $X$.

Definition 3.7 Consider a graph $G=(V, E)$. Let $F$ be a nonempty subset of $E, U$ the set of vertices incident with $F$, and $H=(U, F)$ the subgraph of $G$ induced by $F$. We say that $F$ is independent if, for any subset $X$ of $U$ having more than two points, we have

$$
i_{H}(X) \leq 2|X|-3 .
$$

The empty set is also defined to be independent. All other subsets of $E$ are said to be dependent.

The rigidity matroid $\mathcal{M}(G)=(E, \mathcal{I})$ is defined on the edge set of $G$ by

$$
\mathcal{I}=\{F \subseteq E: F \text { is independent in } G\} .
$$

If every proper subset of $E$ is independent and $|E|=2|V|-2$, then $G$ is called a generic circuit.

Now we define two operations on graphs.

Let $G$ be a graph, and $v_{1} v_{2}$ an edge of $G$. An extension of $G$ along $v_{1} v_{2}$ is obtained from $G$ by subdividing the edge $v_{1} v_{2}$ by a new vertex $v$ and adding a new edge $v w$ connecting $v$ to some vertex other than $v_{1}$ and $v_{2}$.

For two graphs $G_{1}=\left(V_{1}, E_{1}\right)$ and $G_{2}=\left(V_{2}, E_{2}\right)$ with two designated edges $v_{1} w_{1}$ and $v_{2} w_{2}$, the 2-sum of $G_{1}$ and $G_{2}$ along the edge pair $v_{1} w_{1}$ and $v_{2} w_{2}$ is the graph obtained from $G_{1}-v_{1} w_{1}$ and $G_{2}-v_{2} w_{2}$ by identifying $v_{1}$ with $v_{2}$ and $w_{1}$ with $w_{2}$.

The following theorem is similar to atom decomposition.

Theorem 3.8 (Berg and Jordán [2]) A graph $G=(V, E)$ is a generic circuit if and only if $G$ is a connected graph obtained from disjoint copies of $K_{4}$ 's by taking 2-sums and applying extensions.

This theorem was conjectured by R. Connelly in the 1980s and was then proved by Berg and Jordán [2]. For the application to rigidity, we refer to Sect. 6 of that paper.

Remark 3.9 There is a difference between the sum of tensegrities that have a common edge and the 2-sum of graphs, since in a 2-sum we remove the common edge, while for the sum of tensegrities, this edge may only be removed if its resulting edgestress is zero. Therefore an atom decomposition works for graphs that are not generic circuits as well.

\subsection{Calculation of Tensegrity $d$-Characteristic in the Simplest Cases}

We start this subsection with the formulation of a problem, though we do not know the complete solution of it. 
Problem 1 Give a general formula for $\tau_{d}(G)$ in terms of the combinatorics of the graph.

Let us calculate the $d$-TC for a complete graph; this will give us the maximal value of the $d$-TC for fixed number of vertices $n$ and dimension $d$.

Proposition 3.10 For any positive integers $n$ and $d$ satisfying $n \geq d+2$, we have

$$
\tau_{d}\left(K_{n}\right)=\frac{(n-d-1)(n-d)}{2} .
$$

Proof We work by induction on $n$. For $n=d+2$, the $d$-TC equals 1 , as mentioned above. For $n>d+2$, we choose any point configuration $P$ on $n$ points such that no $d+1$ of them lie in a hyperplane. Take $p \in P$. Any tensegrity $\left(K_{n}(P), w\right)$ can be decomposed as a sum of $n-d-1$ atoms with $p$ as vertex and a tensegrity on $P \backslash\{p\}$ with underlying graph $K_{n-1}$. Indeed, we can use such atoms to cancel the given edge-stresses on $n-d-1$ edges at $p$. Then there are only $d$ edges left, so by Lemma 3.5 the edge-stresses on these edges equal zero. We conclude by induction that

$$
\tau_{d}\left(K_{n}\right)=\tau_{d}\left(K_{n-1}\right)+n-d-1=\frac{(n-d-1)(n-d)}{2} .
$$

Now we show how the $d$-TC behaves when we remove an edge of the graph.

Proposition 3.11 Let $G$ be some graph satisfying $\tau_{d}(G)>1$. Let a graph $G^{\prime}$ be obtained from the graph $G$ by erasing one edge. Then

$$
\tau_{d}(G)-\tau_{d}\left(G^{\prime}\right) \in\{0,1\} .
$$

Proof Erasing one edge is equivalent to adding a new linear equation $w_{i, j}=0$ to the linear system defining the space $W(G, P)$ for the graph $G$ (for any point $P$ ). This implies that the space of solutions coincides with $W(G, P)$ or it is a hyperplane in $W(G, P)$.

So, first, $\tau_{d}\left(G^{\prime}\right) \leq \tau_{d}(G)$.

Secondly, since $\tau_{d}(G)=\operatorname{dim}\left(W\left(G, P_{0}\right)\right)$ for some framework $P_{0}$ of a codimension 0 stratum for $G$ (and therefore it belongs to a codimension 0 stratum for $G^{\prime}$ ), we have

$$
\tau_{d}\left(G^{\prime}\right)=\operatorname{dim}\left(W\left(G^{\prime}, P_{0}\right)\right) \geq \operatorname{dim}\left(W\left(G, P_{0}\right)\right)-1=\tau_{d}(G)-1 .
$$

This completes the proof.

As we show in the example below, erasing an edge does not always reduce the tensegrity characteristic.

Example 3.12 Consider the graph shown in Fig. 3. Assume that this graph underlies a tensegrity. Then we can add an atom on the four leftmost vertices, to cancel the edge-stress on edge $f$ for instance. This automatically cancels the edge-stresses on 
Fig. 3 The edge-stress on the edge $e$ is always zero

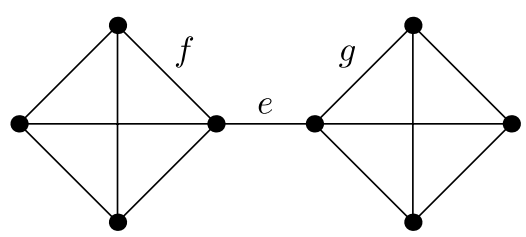

the edges connecting the four leftmost vertices by Lemma 3.5. We can do the same on the right. So the edge-stress on $e$ is zero as well. Therefore the edge-stress on $e$ was zero from the beginning, and hence deleting $e$ does not change the 2-TC. In Example 6.3 we give a less trivial example of this phenomenon.

Let us formulate two general corollaries of Proposition 3.11.

Corollary 3.13 Let $G$ be a graph on $n$ vertices, and $m \in \mathbb{Z}_{>0}$. If $G$ has

$$
m+\frac{n(n-1)}{2}-\tau_{d}\left(K_{n}\right)=m+d n-\frac{d^{2}+d}{2}
$$

edges, then $\tau_{d}(G) \geq m$.

Proof Combine Proposition 3.10 and Proposition 3.11.

The following corollary is useful for the calculation of the tensegrity $d$-characteristic. In Sect. 6.1 we use it to calculate all the tensegrity 2-characteristics for sufficiently connected graphs with less than 8 vertices.

Corollary 3.14 Let $G$ be a graph on $n$ vertices with $\tau_{d}(G) \geq 0$. Assume that $G$ has

$$
d n-\frac{d^{2}+d}{2}+\tau_{d}(G)
$$

edges. Then for any graph $H$ that can be obtained from $G$ by adding $N$ edges, we have

$$
\tau_{d}(H)=\tau_{d}(G)+N
$$

Proof We delete $\tau_{d}\left(K_{n}\right)-\tau_{d}(G)-N$ edges from $K_{n}$ to reach $H$. If the $d$-TC does not drop by 1 at one of these steps, then we apply Proposition 3.11 an additional $N$ times to $H$ to reach $G$. This leads to a wrong value of $\tau_{d}(G)$. So the $d$-TC drops by one in each of the first $\tau_{d}\left(K_{n}\right)-\tau_{d}(G)-N$ steps, and the formula for $\tau_{d}(H)$ follows.

Example 3.15 A pseudo-triangle is a planar polygon with exactly three vertices at which the angles are less than $\pi$. Let $G$ be a planar graph with $n$ vertices and $k$ edges that admits a pseudo-triangular embedding $G(P)$ in the plane, i.e., a noncrossing embedding such that the outer face is convex and all interior faces are pseudo-triangles. It is obvious that a pseudo-triangular embedding $G(P)$ belongs to a codimension 0 stratum of $B_{2}(G)$. By Lemma 2 of [17] we find that: 
- $\tau_{2}(G)=k-(2 n-3)$ if $k-(2 n-3) \geq 1$;

- $\tau_{2}(G) \leq 0$ if $k-(2 n-3)=0$.

(Note that for pseudo-triangular embeddings, we always have $k \geq 2 n-3$.)

\section{Surgeries on Graphs that Preserve the Dimension of the Fibers}

In this section we describe operations that one can perform on a graph without changing the dimensions of the corresponding fibers for the frameworks. We refer to such operations as surgeries. The first type of surgeries is for general dimension, while the other two are restricted to dimension $d=2$. We do not know other similar operations that are not compositions of the surgeries described below.

The idea of surgeries is analogous to the idea of Reidemeister moves in knot theory. If two graphs are connected by a sequence of surgeries, then one obtains tensegrities for the first graph from tensegrities for the second graph and vice versa.

We essentially use surgeries to calculate the list of geometric conditions for the strata for (sufficiently connected) graphs with less than 9 vertices and with zero 2-TC in Sect. 6.2.

\subsection{General Surgeries in Arbitrary Dimension}

For an edge $e$ of a graph $G$, we denote by $G_{e}$ the graph obtained from $G$ by removing $e$.

Denote by $\Sigma_{d}(G)$ the union of codimension zero strata in $B_{d}(G)$. Let $G$ be a graph, and $H$ a subgraph. Consider the map that takes a framework for $G$ to the framework for $H$ by forgetting all the vertices and edges of $G$ that are not in $H$. Denote by $\Sigma_{d}(G, H)$ the preimage of $\Sigma_{d}(H)$ for this map.

Proposition 4.1 (Edge exchange) Let $G$ be a graph, and $H$ an induced subgraph with $\tau_{d}(H)=1$. Consider a configuration $P_{0}$ lying in $\Sigma_{d}(G, H)$. Suppose that there exists a self-stress on the framework $G\left(P_{0}\right)$ that has nonzero edge-stresses for all edges of $H$ and zero edge-stresses on the other edges. Let $e_{1}, e_{2}$ be edges of $H$. Then for any $P \in \Sigma_{d}(G, H)$, we have

$$
W\left(G_{e_{1}}, P\right) \cong W\left(G_{e_{2}}, P\right) .
$$

The corresponding surgery takes the graph $G_{e_{1}}$ to $G_{e_{2}}$ or vice versa.

Remark 4.2 We always have the inclusion $\Sigma_{d}(G) \subset \Sigma_{d}(G, H)$, which follows directly from the definition of the strata. Nevertheless the set $\Sigma_{d}(G, H)$ usually contains many strata of $B_{d}(G)$ of positive codimension. So Proposition 4.1 is applicable to all strata of codimension zero and to some strata of positive codimension.

For the proof of Proposition 4.1, we need the following lemma. 
Lemma 4.3 Let $G$ be a graph with $\tau_{d}(G)=1$, and e one of its edges. Suppose that there exists a configuration $P_{0} \in \Sigma_{d}(G)$ and a nonzero self-stress $w_{0}$ such that $w_{0}(e)=0$. Then for any tensegrity $(G(P), w)$ with $P \in \Sigma_{d}(G)$, we get $w(e)=0$.

Proof Since $\tau_{d}(G)=1$ and $P_{0} \in \Sigma_{d}(G)$, any tensegrity $\left(G\left(P_{0}\right), w\right)$ satisfies the condition $w(e)=0$. Therefore, any tensegrity with $P$ in the same stratum as $P_{0}$ has zero edge-stress at $e$. So the condition always to have zero edge-stress at e defines a somewhere dense subset $S$ in $B_{d}(G)$. Since the condition is defined by a solution of a certain linear system, $S$ is dense in $B_{d}(G)$. It follows that $\Sigma_{d}(G)$ is a subset of $S$.

Proof of Proposition 4.1 From Lemma 4.3 we have that for any configuration of $\Sigma_{d}(H)$, there exists a unique up to a scalar self-stress that is nonzero at each edge of $H$. The uniqueness follows from the fact that $\tau_{d}(H)=1$. Hence, for any configuration of $\Sigma_{d}(G, H)$, there exists a unique up to a scalar self-stress that is nonzero at each edge of $H$ and zero at all other edges of $G$.

For any $P \in \Sigma_{d}(G, H)$, we obtain an isomorphism between $W\left(G_{e_{1}}, P\right)$ and $W\left(G_{e_{2}}, P\right)$ by adding the unique tensegrity on the underlying subgraph $H$ of $G$ that cancels the edge-stress on $e_{2}$, considered as edge of $G_{e_{1}}$.

In particular one can use atoms (i.e., $H=K_{d+2}$ ) in the above proposition.

Corollary 4.4 In the notation and with the conditions of Proposition 4.1, we have: if either $\tau_{d}\left(G_{e_{1}}\right)>0$ or $\tau_{d}\left(G_{e_{2}}\right)>0$, then

$$
\tau_{d}\left(G_{e_{1}}\right)=\tau_{d}\left(G_{e_{2}}\right) .
$$

Proof The statement follows directly from Proposition 4.1 and Corollary 3.4.

Let us show how to use the above corollary to compute the tensegrity characteristic.

Example 4.5 We calculate the 2-TC of the graph $G_{0}$ shown in Fig. 4. Consider the atom $H$ on the vertices $v_{1}, v_{2}, v_{3}$, and $v_{4}$ and let $e_{1}, e_{2}$ be the edges $v_{2} v_{4}, v_{1} v_{3}$,

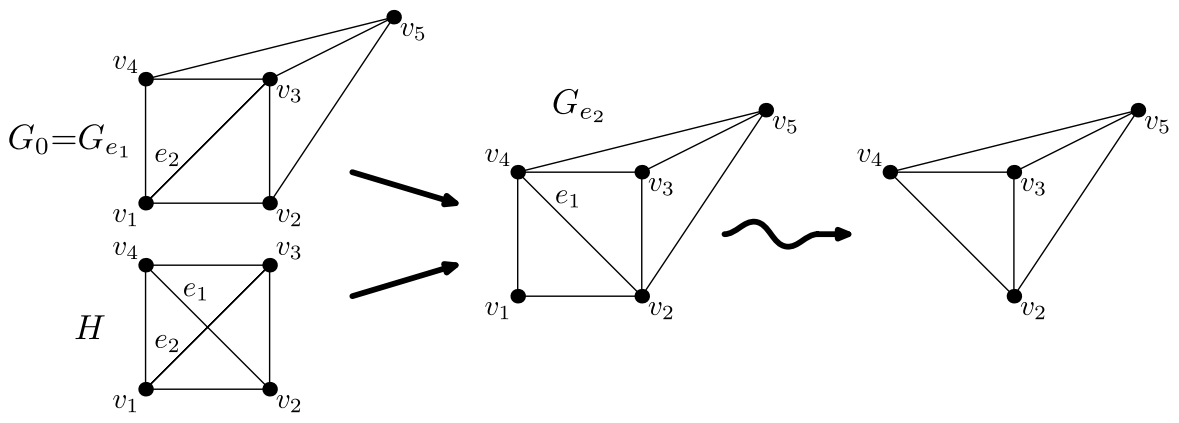

Fig. 4 This shows that $\tau_{2}\left(G_{0}\right)=1$ 


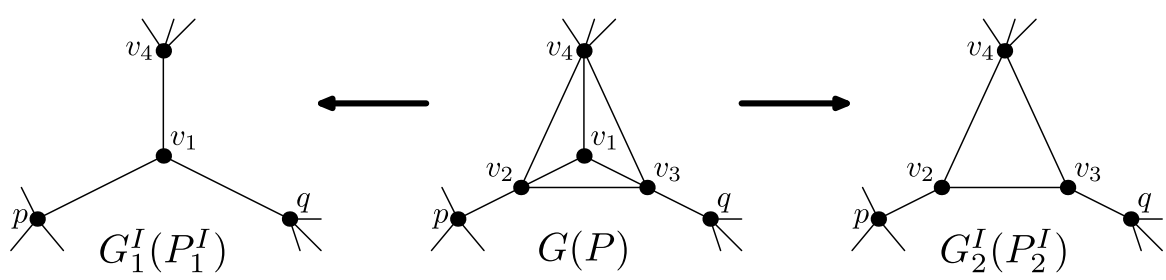

Fig. 5 Surgery $I$

respectively. Denote by $G$ the graph obtained from $G_{0}$ by adding the edge $e_{2}$. So the graph $G_{0}$ is actually $G_{e_{1}}$. By Corollary 3.13 we have $\tau_{2}\left(G_{0}\right) \geq 1$, and hence it is possible to apply Corollary 4.4. Consider the graph $G_{e_{2}}$; it is shown in Fig. 4 in the middle. The degree of the vertex $v_{1}$ in this graph equals 2, so by Lemma 3.5 the edge-stresses on its incoming edges equal zero if the points $v_{1}, v_{2}$, and $v_{4}$ are not on a line. After removing these two edges and the vertex $v_{1}$, we get the graph of an atom. Therefore,

$$
\tau_{2}\left(G_{0}\right)=1
$$

\subsection{Additional Surgeries in Dimension Two}

In this subsection we study two surgeries on edges of plane frameworks that do not change the dimension of the fibers of the frameworks.

Surgery $I$ Consider a graph $G$ and a framework $G(P)$. Let $G$ contain the complete graph $K_{4}$ with vertices $v_{1}, v_{2}, v_{3}$, and $v_{4}$ as an induced subgraph. Suppose that the edges between $v_{1}, v_{2}, v_{3}, v_{4}$ and other vertices of $G$ are as follows:

- $p v_{2}$ and $q v_{3}$ for unique vertices $p$ and $q$;

- the edges $p v_{1}$ and $q v_{1}$;

- any set of edges from $v_{4}$.

In addition we require that the framework $G(P)$ has the triples of points $\left(p, v_{1}, v_{2}\right)$ and $\left(q, v_{1}, v_{3}\right)$ on one line. See Fig. 5 in the middle.

Let us delete from the graph $G$ the vertices $v_{2}$ and $v_{3}$ (the vertex $v_{1}$ ) with all edges adjacent to them. We denote the resulting graph by $G_{1}^{I}$ (by $G_{2}^{I}$, respectively). The corresponding framework is denoted by $G_{1}^{I}\left(P_{1}^{I}\right)$ (by $G_{2}^{I}\left(P_{2}^{I}\right)$, respectively). See Fig. 5 on the left (on the right). Surgery $I$ takes $G_{1}^{I}$ to $G_{2}^{I}$ or vice versa.

Remark 4.6 This surgery looks like the $\Delta Y$ exchange in matroid theory, but it is not exactly the same. For instance, to go from $G_{2}^{I}\left(P_{2}^{I}\right)$ to $G_{1}^{I}\left(P_{1}^{I}\right)$ in Fig. 5, we basically replace the triangle by a 3 -star, but we assume here that the triangle has two vertices of degree 3 .

Proposition 4.7 Consider the frameworks $G(P), G_{1}^{I}\left(P_{1}^{I}\right)$, and $G_{2}^{I}\left(P_{2}^{I}\right)$ as above. If the triples of points $\left(p, v_{2}, v_{3}\right),\left(q, v_{2}, v_{3}\right),\left(p, v_{2}, v_{4}\right),\left(q, v_{3}, v_{4}\right)$, and $\left(v_{2}, v_{3}, v_{4}\right)$ 
are not on a line, then we have

$$
W\left(G_{1}^{I}, P_{1}^{I}\right) \cong W\left(G_{2}^{I}, P_{2}^{I}\right)
$$

Proof We explain how to go from $W\left(G_{2}^{I}, P_{2}^{I}\right)$ to $W\left(G_{1}^{I}, P_{1}^{I}\right)$. The inverse map is simply given by the reverse construction. By the conditions the intersection point $v_{1}$ of $p v_{2}$ and $q v_{3}$ is uniquely defined and not on the lines through $v_{2}$ and $v_{4}$, or $v_{3}$ and $v_{4}$. We add the uniquely defined atom on $v_{1}, v_{2}, v_{3}, v_{4}$ to $G_{2}^{I}\left(P_{2}^{I}\right)$ that cancels the edge-stress on $v_{2} v_{3}$. Since $p, v_{2}, v_{1}$ lie on one line, this surgery also cancels the edge-stress on $v_{2} v_{4}$ and similarly for $v_{3} v_{4}$. Due to the equilibrium condition at $v_{2}$, we can replace the edges $p v_{2}$ and $v_{2} v_{1}$ with their edge-stresses $w_{p, 2}$ and $w_{2,1}$ by an edge $p v_{1}$ with edge-stress $w_{p, 1}$ defined by one of the following vector equations:

$$
w_{p, 2}\left(v_{2}-p\right)=w_{p, 1}\left(v_{1}-p\right)=w_{2,1}\left(v_{1}-v_{2}\right)
$$

This uniquely defines a self-stress on $G_{1}^{I}\left(P_{1}^{I}\right)$.

Corollary 4.8 Assume that one of the following conditions holds:

(1) $\tau_{2}\left(G_{1}^{I}\right)>0$ or $\tau_{2}\left(G_{2}^{I}\right)>0$.

(2) $\tau_{2}\left(G_{1}^{I}\right)=0$, and there is a codimension 1 stratum $S$ of $B_{2}\left(G_{1}^{I}\right)$ such that:

- $\operatorname{dim} W\left(G_{1}^{I}, P\right)>0$ for a $G_{1}^{I}(P)$ in the stratum $S$;

- the stratum $S$ is not contained in the subset of $B_{2}\left(G_{1}^{I}\right)$ of frameworks having one of the triples of points $\left(p, v_{1}, q\right),\left(p, v_{1}, v_{4}\right)$, or $\left(q, v_{1}, v_{4}\right)$ on one line.

(3) $\tau_{2}\left(G_{2}^{I}\right)=0$, and there is a codimension 1 stratum $S^{\prime}$ of $B_{2}\left(G_{2}^{I}\right)$ such that:

- $\operatorname{dim} W\left(G_{2}^{I}, P^{\prime}\right)>0$ for a $G_{2}^{I}\left(P^{\prime}\right)$ in the stratum $S^{\prime}$;

- the stratum $S^{\prime}$ is not contained in the subset of $B_{2}\left(G_{2}^{I}\right)$ of frameworks having $\left(p, v_{2}, v_{3}\right),\left(q, v_{2}, v_{3}\right),\left(p, v_{2}, v_{4}\right),\left(q, v_{3}, v_{4}\right)$, or $\left(v_{2}, v_{3}, v_{4}\right)$ on one line.

Then

$$
\tau_{2}\left(G_{1}^{I}\right)=\tau_{2}\left(G_{2}^{I}\right)
$$

Proof Let $A$ be the subset of $B_{2}\left(G_{2}^{I}\right)$ of frameworks having $\left(p, v_{2}, v_{3}\right),\left(q, v_{2}, v_{3}\right)$, $\left(p, v_{2}, v_{4}\right),\left(q, v_{3}, v_{4}\right)$, or $\left(v_{2}, v_{3}, v_{4}\right)$ on one line. Let $B$ be the subset of $B_{2}\left(G_{1}^{I}\right)$ of frameworks having $\left(p, v_{1}, q\right),\left(p, v_{1}, v_{4}\right)$, or $\left(q, v_{1}, v_{4}\right)$ on one line. Note that $A$ and $B$ are of codimension 1. The proof of Proposition 4.7 gives a surjective map

$$
\varphi: B_{2}\left(G_{2}^{I}\right) \backslash A \rightarrow B_{2}\left(G_{1}^{I}\right) \backslash B
$$

inducing an isomorphism between the linear fibers above $G(P) \in B_{2}\left(G_{2}^{I}\right) \backslash A$ and $\varphi(G(P))$. Now in all the cases (1)-(3) the statement of the corollary follows directly from the definition of the tensegrity characteristic. 


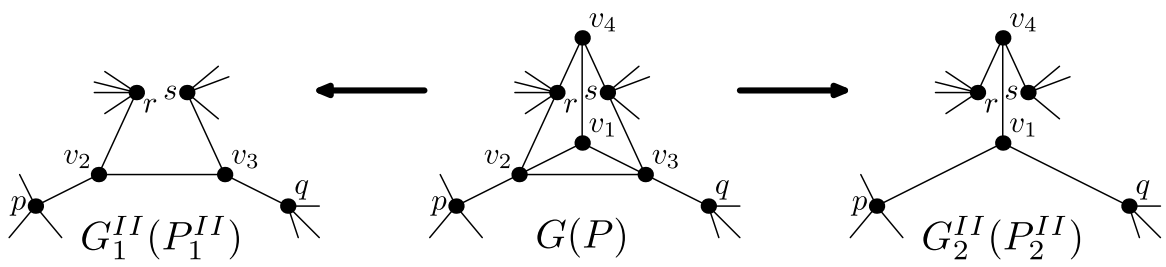

Fig. 6 Surgery $I I$

Surgery II Consider a graph $G$ and a framework $G(P)$. Let $G$ contain the complete graph $K_{4}$ with vertices $v_{1}, v_{2}, v_{3}$, and $v_{4}$ as an induced subgraph. Suppose that the set of edges between $v_{1}, v_{2}, v_{3}, v_{4}$ and other vertices of $G$ is

$$
\left\{p v_{1}, p v_{2}, q v_{1}, q v_{3}, r v_{2}, r v_{4}, s v_{3}, s v_{4}\right\}
$$

for unique points $p, q, r, s$. In addition we require that the framework $G(P)$ has the triples of points

$$
\left(p, v_{1}, v_{2}\right), \quad\left(q, v_{1}, v_{3}\right), \quad\left(r, v_{2}, v_{4}\right), \quad \text { and } \quad\left(s, v_{3}, v_{4}\right)
$$

on one line. See Fig. 6 in the middle.

Let us delete from the graph $G$ the vertices $v_{1}$ and $v_{4}\left(v_{2}\right.$ and $\left.v_{3}\right)$ with all edges adjacent to them. We denote the resulting graph by $G_{1}^{I I}$ (by $G_{2}^{I I}$, respectively). The corresponding framework is denoted by $G_{1}^{I I}\left(P_{1}^{I I}\right)$ (by $G_{2}^{I I}\left(P_{2}^{I I}\right)$, respectively). See Fig. 6 on the left (on the right). Surgery $I I$ takes $G_{1}^{I I}$ to $G_{2}^{I I}$ or vice versa.

The proofs of the proposition and corollary below are similar to the proofs of Proposition 4.7 and Corollary 4.8 .

Proposition 4.9 Consider the frameworks $G(P), G_{1}^{I I}\left(P_{1}^{I I}\right)$, and $G_{2}^{I I}\left(P_{2}^{I I}\right)$ as above. If none of the triples of points $\left(p, q, v_{1}\right),\left(p, v_{1}, v_{4}\right),\left(r, v_{1}, v_{4}\right),\left(q, v_{1}, v_{4}\right)$, $\left(s, v_{1}, v_{4}\right)$, or $\left(r, s, v_{4}\right)$ lie on a line, then we have

$$
W\left(G_{1}^{I I}, P_{1}^{I I}\right) \cong W\left(G_{2}^{I I}, P_{2}^{I I}\right) .
$$

Corollary 4.10 Assume that one of the following conditions holds:

(1) $\tau_{2}\left(G_{1}^{I I}\right)>0$ or $\tau_{2}\left(G_{2}^{I I}\right)>0$.

(2) $\tau_{2}\left(G_{1}^{I I}\right)=0$, and there is a codimension 1 stratum $S$ of $B_{2}\left(G_{1}^{I I}\right)$ such that:

- $\operatorname{dim} W\left(G_{1}^{I I}, P\right)>0$ for a $G_{1}^{I I}(P)$ in the stratum $S$;

- the stratum $S$ is not contained in the subset of $B_{2}\left(G_{1}^{I I}\right)$ of frameworks having $\left(p, v_{2}, v_{3}\right),\left(q, v_{2}, v_{3}\right),\left(p, v_{2}, r\right),\left(q, v_{3}, s\right),\left(r, v_{2}, v_{3}\right)$, or $\left(s, v_{2}, v_{3}\right)$ on one line.

(3) $\tau_{2}\left(G_{2}^{I I}\right)=0$, and there is a codimension 1 stratum $S^{\prime}$ of $B_{2}\left(G_{2}^{I I}\right)$ such that:

- $\operatorname{dim} W\left(G_{2}^{I I}, P^{\prime}\right)>0$ for a $G_{2}^{I I}\left(P^{\prime}\right)$ in the stratum $S^{\prime}$; 
- the stratum $S^{\prime}$ is not contained in the subset of $B_{2}\left(G_{2}^{I I}\right)$ of frameworks having $\left(p, q, v_{1}\right),\left(p, v_{1}, v_{4}\right),\left(r, v_{1}, v_{4}\right),\left(q, v_{1}, v_{4}\right),\left(s, v_{1}, v_{4}\right)$, or $\left(r, s, v_{4}\right)$ on one line.

Then

$$
\tau_{2}\left(G_{1}^{I I}\right)=\tau_{2}\left(G_{2}^{I I}\right)
$$

\section{Geometric Relations for Strata and Complexity of Tensegrities in the Two-Dimensional Case}

In all the observed examples of plane tensegrities with a given graph, the strata for which a tensegrity is realizable are defined by certain geometric conditions on the points of the corresponding frameworks. In this section we study such geometric conditions. In Sect. 5.1 we describe an example of a geometric condition for a particular graph. Further, in Sect. 5.2 we give general definitions related to systems of geometric conditions. Finally, in Sects. 5.3 and 5.4 we state theorems and formulate open questions related to the geometric nature of tensegrity strata.

To avoid problems with describing annoying cases of parallel/nonparallel lines, we extend the plane $\mathbb{R}^{2}$ to the projective space. It is convenient for us to consider the following model of the projective space: $\mathbb{R} P^{2}=\mathbb{R}^{2} \cup l_{\infty}$. The set of points $l_{\infty}$ is the set of all "directions" in the plane. The set of lines of $\mathbb{R} P^{2}$ is the set of all plane lines (each plane line contains now a new point of $l_{\infty}$ that is the direction of $l$ ) together with the line $l_{\infty}$. Now any two lines intersect at exactly one point.

\subsection{A Simple Example}

First, we study the graph shown in Fig. 2 on the left and denote it by $G_{0}$. White and Whiteley [25] proved that the 2-TC of this graph is zero. They showed that there exists a nonzero tensegrity with graph $G_{0}$ and framework $P$ iff the points of $P$ satisfy one of the following three conditions:

(i) the lines $v_{1} v_{2}, v_{3} v_{4}$, and $v_{5} v_{6}$ have a common nonempty intersection (in $\mathbb{R} P^{2}$ );

(ii) the vertices $v_{1}, v_{4}$, and $v_{5}$ are in one line;

(iii) the vertices $v_{2}, v_{3}$, and $v_{6}$ are in one line.

We remind that the base $B\left(G_{0}\right)$ of the configuration space is $\mathbb{R}^{12}$ with coordinates $\left(x_{1}, y_{1}, \ldots, x_{6}, y_{6}\right)$, where $\left(x_{i}, y_{i}\right)$ are the coordinates of $v_{i}$. Condition (i) defines a degree 4 hypersurface with equation

$$
\operatorname{det}\left(\begin{array}{ccc}
y_{1}-y_{2} & y_{3}-y_{4} & y_{5}-y_{6} \\
x_{2}-x_{1} & x_{4}-x_{3} & x_{6}-x_{5} \\
x_{1} y_{2}-x_{2} y_{1} & x_{3} y_{4}-x_{4} y_{3} & x_{5} y_{6}-x_{6} y_{5}
\end{array}\right)=0,
$$

and conditions (ii) and (iii) define the conics

$$
\begin{aligned}
& x_{1} y_{4}+x_{4} y_{5}+x_{5} y_{1}-x_{1} y_{5}-x_{4} y_{1}-x_{5} y_{4}=0 \quad \text { and } \\
& x_{2} y_{3}+x_{3} y_{6}+x_{6} y_{2}-x_{2} y_{6}-x_{3} y_{2}-x_{6} y_{3}=0
\end{aligned}
$$

respectively. 


\subsection{Systems of Geometric Conditions}

First we introduce two notations as in the Cayley algebra (for basic introduction to the Cayley algebra, also called Grassmann or double algebra, we refer to [1, 11, 23]). Denote a line through the points $p$ and $q$ by $p \vee q$, or $p q$ for short. For two lines $l_{1}$ and $l_{2}$, we denote by $l_{1} \wedge l_{2}$ the intersection point of the lines.

Let us define the following three elementary geometrical conditions. Consider an ordered subset $P=\left\{p_{1}, \ldots, p_{n}\right\}$ of the projective plane:

- 2-point condition. We say that the subset $P$ satisfies the condition $p_{i}=p_{j}$ if $p_{i}$ coincides with $p_{j}$.

- 3-point condition. We say that the subset $P$ satisfies the condition

$$
p_{i} \nabla p_{j} \nabla p_{k}=0
$$

if the points $p_{i}, p_{j}$, and $p_{k}$ are on a line.

- 5-point condition. We say that the subset $P$ satisfies the condition

$$
p_{i}=\left[p_{j}, p_{j^{\prime}} ; p_{k}, p_{k^{\prime}}\right]
$$

if the four points $p_{j}, p_{j^{\prime}}, p_{k}$, and $p_{k^{\prime}}$ are on a line and $p_{i}$ also belongs to this line, or if $p_{i}=\left(p_{j} \vee p_{j^{\prime}}\right) \wedge\left(p_{k} \vee p_{k^{\prime}}\right)$.

Note that we give the last definition in terms of closures, since $p q \wedge r s$ is not defined for all 4-tuples, but rather for a dense subset.

Definition 5.1 Consider a system of elementary geometric conditions for ordered $n$-point subsets of $\mathbb{R} P^{2}$, and let $m \leq n$ :

- We say that the ordered $n$-point subset $P$ of projective plane satisfies the system of elementary geometric conditions if $P$ satisfies each of these conditions.

- We say that the ordered subset $\left\{p_{1}, \ldots, p_{m}\right\}$ satisfies conditionally the system of elementary geometric conditions if there exist points $q_{1}, \ldots, q_{n-m}$ such that the ordered set

$$
\left\{p_{1}, \ldots, p_{m}, q_{1}, \ldots, q_{n-m}\right\}
$$

satisfies the system. We call the number $n-m$ the conditional number of the system.

Example 5.2 The condition that six points $p_{1}, \ldots, p_{6}$ lie on a conic is equivalent to the following geometric conditional system:

$$
\left\{\begin{array}{l}
q_{1}=\left[p_{1}, p_{2} ; p_{4}, p_{5}\right], \\
q_{2}=\left[p_{2}, p_{3} ; p_{5}, p_{6}\right], \\
q_{3}=\left[p_{3}, p_{4} ; p_{1}, p_{6}\right], \\
q_{1} \nabla q_{2} \nabla q_{3}=0 .
\end{array}\right.
$$

This is a reformulation of Pascal's theorem. The conditional number is 3 here.

For short, we can rewrite the system as follows:

$$
\left(p_{1} p_{2} \wedge p_{4} p_{5}\right) \nabla\left(p_{2} p_{3} \wedge p_{5} p_{6}\right) \nabla\left(p_{3} p_{4} \wedge p_{1} p_{6}\right)=0 .
$$


Example 5.3 The condition for six points $p_{1}, \ldots, p_{6}$ that the lines $p_{1} p_{2}, p_{3} p_{4}$, and $p_{5} p_{6}$ have a common point is equivalent to the following geometric conditional system:

$$
\left\{\begin{array}{l}
q_{1}=\left[p_{1}, p_{2} ; p_{3}, p_{4}\right], \\
q_{1} \nabla p_{5} \nabla p_{6}=0,
\end{array}\right.
$$

or in a shorter form,

$$
\left(p_{1} p_{2} \wedge p_{3} p_{4}\right) \nabla p_{5} \nabla p_{6}=0 .
$$

The conditional number of the system is 1 .

\subsection{Conjecture on Geometric Structure of the Strata}

For a given positive integer $k$ and a graph $G$, consider the set of all frameworks $G(P)$ at which the dimension of the fiber $W(G, P)$ is greater than or equal to $k$. We call this set the $(G, k)$-stratum. Since any $(G, k)$-stratum is a finite union of strata of the base $B_{2}(G)$, it is semialgebraic.

Definition 5.4 Let $G$ be a graph, and $k$ be a positive integer. The $(G, k)$-stratum is said to be geometric if it is a finite union of the sets of conditional solutions of systems of geometric conditions (in these systems $p_{1}, \ldots, p_{m}$ correspond to the vertices of the graph).

Theorem 5.5 For any graph and any integer $k$, the $(G, k)$-stratum is geometric.

This result was communicated to us by W. Whiteley.

Proof The $(G, k)$-stratum is defined as the set of frameworks $G(P)$ in $B_{2}(G)$ such that $\operatorname{dim} W(G, P) \geq k$. As explained in Sect. 2.2, this corresponds to the locus where the rigidity matrix $M(G(P))$ has rank less than $|E|-k$, where $E$ is the edge set of $G$. So the $(G, k)$-stratum is the set of frameworks where the sum of the squares of all $(|E|-k+1)$-minors of the rigidity matrix vanishes, and hence it is described by a polynomial $f$ in the coordinates of the points of $P$. According to the proof of Theorem 5.10 of [18], the rank of the rigidity matrix is projectively invariant, and hence $f$ is an invariant as in Sect. 2 of [25]. The first fundamental theorem of invariant theory [11] shows then that $f$ can be expressed as a bracket algebra polynomial $g$. The vanishing of $g$ is equivalent to the vanishing of a Cayley algebra expression (see Theorem 1 of [23]), and this proves the theorem.

The "inverse problem" is still open:

Problem 2 Suppose we are given a certain system of geometric conditions. How to verify if this system defines some $(G, k)$-strata? How to find all the graphs $G$ corresponding to these strata?

Finally, we formulate a problem for nonplanar tensegrities. 
Problem 3 Find analogous elementary geometric conditions in the three- and higherdimensional cases.

We refer to [25] for examples of geometric conditions in dimension 3.

\subsection{Complexity of the Strata}

We end this section with a discussion of the complexity of geometric $(G, k)$-strata.

A geometric $(G, k)$-stratum is defined by some union of the conditional solutions of systems of geometric conditions. Each system in this union has its own conditional number. Take the maximal among all the conditional numbers in the union. We call the minimal number among such maximal numbers for all the unions of systems defining the same $(G, k)$-stratum the geometric complexity of the $(G, k)$-stratum.

Example 5.6 The geometric complexity of the $\left(G_{0}, 1\right)$-stratum for the graph $G_{0}$ described in Sect. 5.1 and shown in Fig. 2 on the left equals 3.

Problem 4 Find the asymptotics of the maximal complexity of geometric $(G, k)$ strata with bounded number of vertices as $k$ tends to infinity.

\section{Plane Tensegrities with a Small Number of Vertices}

In this section we work in the two-dimensional case (unless otherwise stated). In Sect. 6.1 we study the 2-TC of graphs. In particular, we calculate the 2-TC for sufficiently connected graphs with seven or less vertices. In Sect. 6.2 we give a list of geometric conditions for realizability of tensegrities in the plane for graphs with zero 2-TC.

\subsection{On the Tensegrity 2-Characteristic of Graphs}

How to characterize the graphs that have a certain number of vertices, edges, and a given 2-TC? Corollary 2.6 of Laman's theorem gives us an answer for graphs $G=$ $(V, E)$ with $2|V|-|E|=3$ and $\tau_{2}(G)=0$ : they are isostatic. For other cases, not much is known. In this subsection we have a first glance at the general problem.

Recall the following definitions from graph theory. Let $G$ be a graph. The vertex connectivity $\kappa(G)$ is the minimal number of vertices whose deletion disconnects $G$. The edge connectivity $\lambda(G)$ is the minimal number of edges whose deletion disconnects $G$. It is well known that $\kappa(G) \leq \lambda(G)$.

For general dimension $d$, let $G(P)$ be a framework in $\mathbb{R}^{d}$ with underlying graph $G$. If $\kappa(G)<d$ or $\lambda(G)<d+1$, then $G(P)$ consists of two or more pieces that can rotate with respect to each other. So, for us, the most interesting graphs are those with $\kappa(G) \geq d$ and $\lambda(G) \geq d+1$.

Example 6.1 (Proposition) Let $G$ be a 2-vertex and 3-edge connected graph with $k$ edges and $n$ vertices. If $n \leq 7$, then

$$
\tau_{2}(G)=k-2 n+3 .
$$


Fig. 7 A graph $G$ with 9 vertices, 15 edges, and $\tau_{2}(G)=1$
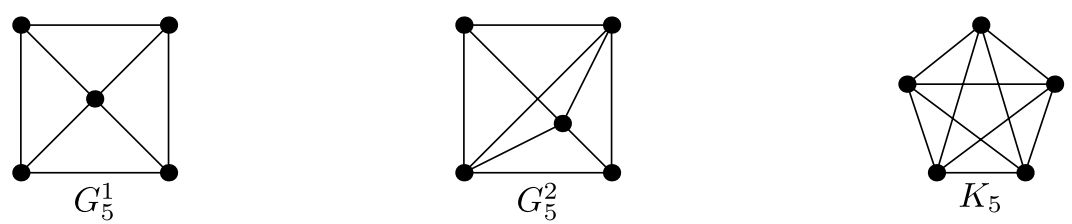

Fig. 8 The three possible graphs with five vertices, $\kappa \geq 2$, and $\lambda \geq 3$
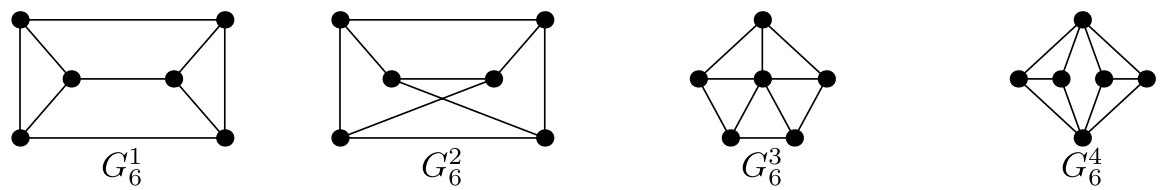

Fig. 9 The four graphs with six vertices, $\kappa \geq 2, \lambda \geq 3$, and a minimal number of edges

Remark 6.2 In particular we have equality in Corollary 3.13 under the conditions of Proposition-Example 6.1. The formula of Proposition-Example 6.1 holds for many graphs in general, see, for instance, Example 3.15. It does not always hold for graphs with 9 vertices as the example below shows.

Example 6.3 Let $G$ be the graph with 9 vertices and 15 edges as in Fig. 7. The graph $G$ satisfies $k=2 n-3$, but it is not isostatic, so $\tau_{2}(G)>0$. In fact it is not hard to prove that $\tau_{2}(G)=1$. So the formula of Proposition-Example 6.1 does not hold for all graphs. Note that $G$ contains the complete graph $K_{4}$ as an induced subgraph, so this implies that the stresses on the edges that do not belong to this $K_{4}$ are zero for a framework in a codimension 0 stratum of $B_{2}(G)$.

Proof of Proposition-Example 6.1 We use a classification argument.

Four vertices. For the complete graph $K_{4}$, we have $\tau_{2}\left(K_{4}\right)=1=6-8+3$. There are no other graphs satisfying the conditions of the proposition.

Five vertices. There are three possibilities, we show them in Fig. 8. From Proposition 3.10 we know that $\tau_{2}\left(K_{5}\right)=3=10-10+3$, and in Example 4.5 we have seen that $\tau_{2}\left(G_{5}^{1}\right)=1=8-10+3$. To see that $\tau_{2}\left(G_{5}^{2}\right)=2$, we apply Corollary 3.14.

Six vertices. From the classification of graphs on six vertices (see, for instance, [22]) we know that any such 2-vertex and 3-edge connected graph can be obtained by adding edges to one of the four graphs shown in Fig. 9. By Corollary 3.14 it suffices to check the formula of the proposition for them.

Note that $G_{6}^{1}$ and $G_{6}^{2}$ are isostatic, so they both have zero 2-TC. For $G_{6}^{3}$, we proceed as follows. From Corollary 3.13 it follows that $\tau_{2}\left(G_{6}^{3}\right) \geq 1$. Then we use Propo- 

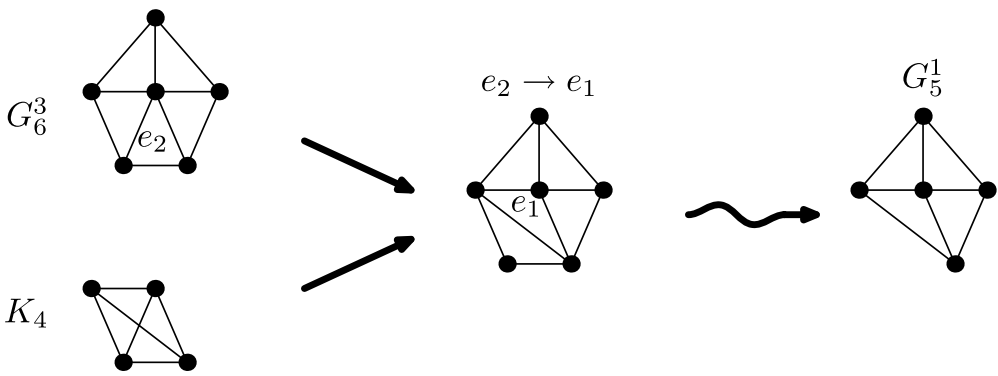

Fig. 10 Using Proposition 4.1, we get that $\tau_{2}\left(G_{6}^{3}\right)=\tau_{2}\left(G_{5}^{1}\right)=1$
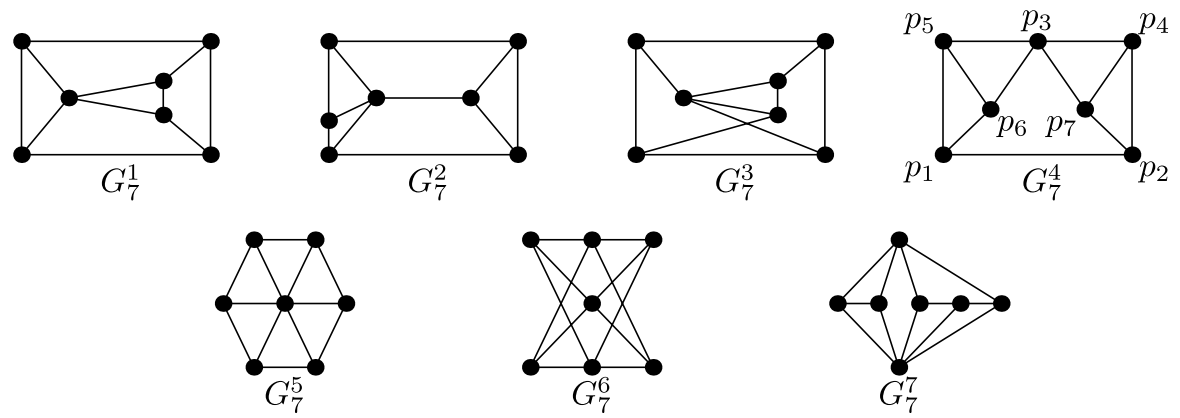

Fig. 11 The seven graphs with seven vertices, $\kappa \geq 2, \lambda \geq 3$, and a minimal number of edges

sition 4.1 in the same way as in Example 4.5 to show that

$$
\tau_{2}\left(G_{6}^{3}\right)=\tau_{2}\left(G_{5}^{1}\right)=1, \quad \text { and again } \quad 10-12+3=1,
$$

see Fig. 10.

It is easy to see that the same argument works to show that $\tau_{2}\left(G_{6}^{4}\right)=1$.

Seven vertices. From the classification of graphs with seven vertices (see [22]) we get that all 2-vertex and 3-edge connected graphs on seven vertices can be obtained by adding edges to one of the seven graphs shown in Fig. 11. By Corollary 3.14 it suffices again to check these graphs.

The graphs $G_{7}^{1}, G_{7}^{2}, G_{7}^{3}$, and $G_{7}^{4}$ are isostatic, so they all have zero 2-TC.

The other three have 12 edges. We apply Corollary 4.8 to $G_{6}^{3}$ and $G_{6}^{4}$ to obtain that

$$
\tau_{2}\left(G_{7}^{5}\right)=1 \quad \text { and } \quad \tau_{2}\left(G_{7}^{7}\right)=1 .
$$

To prove that the 2-TC of $G_{7}^{6}=K_{3,4}$ is 1 , we proceed as follows. First, $\tau_{2}\left(G_{7}^{6}\right) \geq 1$ by Corollary 3.13. Then we apply Proposition 4.1 as shown in Fig. 12. The graph $G$ has 6 vertices and 10 edges, and thus we have $\tau_{2}(G)=1$. It is easy to check that for a general position framework $G(P)$ with a nonzero self-stress, all edges of $G(P)$ have nonzero stress. On the middle picture we get a vertex of degree 2 , so we reduce to the graph $H$ on the right. Note that $H$ is isomorphic to $G$, so $\tau_{2}(H)=1$. Hence $\tau_{2}\left(G_{7}^{6}\right)=1$ as well. 


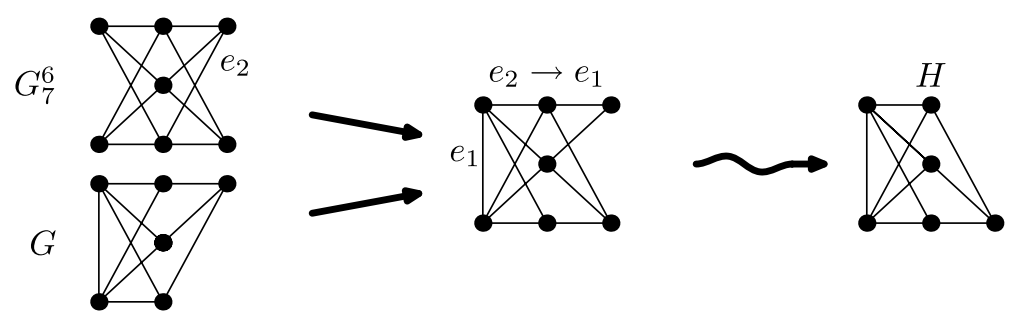

Fig. 12 Using Proposition 4.1, one sees that $\tau_{2}\left(G_{7}^{6}\right)=1$

\subsection{Geometric Conditions for Realizability of Plane Tensegrities for Graphs with Zero Tensegrity 2-Characteristic}

Like in intersection theory of algebraic varieties, it often happens that strata for a graph with negative 2-TC are obtained as intersections of closures of some strata of graphs with zero 2-TC. So the conditions for realizability of plane tensegrities for graphs with zero 2-TC are the most important. In this subsection we give all the conditions for the zero 2-TC graphs with number of vertices not exceeding 8 .

In practice one would like to construct a tensegrity without struts or cables with zero edge-stress. So it is natural to give the following definition. We say that a graph $G$ is visible at the framework $P$ if there exists a self-stress that is nonzero at each edge of this framework.

Remark 6.4 Visibility restrictions remove many degenerate strata. For instance, if a zero 2-TC graph $G$ has a complete subgraph on vertices $v_{1}, v_{2}$, and $v_{3}$, then the codimension 1 stratum defined by the condition that the points $v_{1}, v_{2}$, and $v_{3}$ are on one line does in many cases not contain visible frameworks.

Let us list the geometric conditions for the vertices of all visible 2-vertex and 3 -edge connected graphs with $n$ vertices and zero 2 -TC for $n \leq 8$. To find the geometric conditions, we essentially use the surgeries of Sect. 4 , see Propositions 4.1, 4.7, and 4.9.

In Table 1 we use, besides the elementary, also the following two additional geometric conditions:

- six points are on a conic;

- for six points $p_{1}, \ldots, p_{6}$, the lines $p_{1} p_{2}, p_{3} p_{4}$, and $p_{5} p_{6}$ have a common nonempty intersection.

As we have seen in Examples 5.2 and 5.3, these conditions are equivalent to geometric conditional systems.

Geometric conditions for some of the graphs in Table 1 were known before. For instance, geometric conditions in terms of bracket polynomials for both 6-vertex graphs in the table, for the third 7-vertex graph, and the twenty-seventh 8-valent graph are given by White and Whiteley [25]. For the relation of bipartite graphs (the second 6-vertex graph here) with rectangular bar constructions, see the paper [4] by E.D. Bolker and H. Crapo. 
Remark 6.5 For the last three graphs in the table, we have two distinct equations. Nevertheless, the 2-TC of the graphs are zero. This is similar to the case of noncomplete intersections in algebraic geometry.

\section{Table 1}

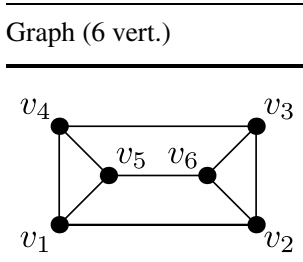

Sufficient geometric conditions

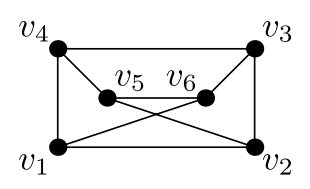

The six points $v_{1}, v_{2}, v_{3}, v_{4}, v_{5}$, and $v_{6}$ are on a conic

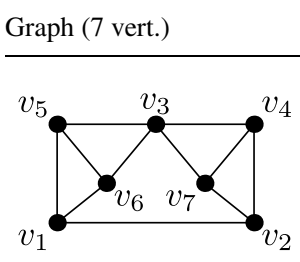

Sufficient geometric conditions

$v_{1} \nabla v_{2} \nabla v_{3}=0$

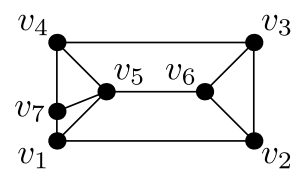

The lines $v_{1} v_{2}, v_{3} v_{4}$, and $v_{5} v_{6}$ have a common nonempty intersection

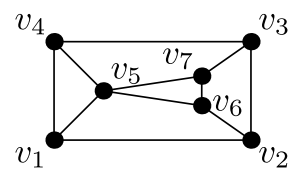

The lines $v_{1} v_{2}, v_{3} v_{4}$, and $v_{5} p$, where $p=\left[v_{2}, v_{6} ; v_{3}, v_{7}\right]$, have a common nonempty intersection

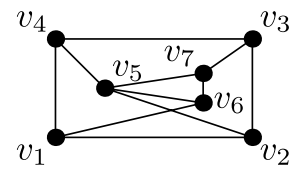

The six points $v_{1}, v_{2}, v_{3}, v_{4}, v_{5}$, and $p$, where $p=\left[v_{1}, v_{6} ; v_{3}, v_{7}\right]$, are on a conic 
Table 1 (Continued)
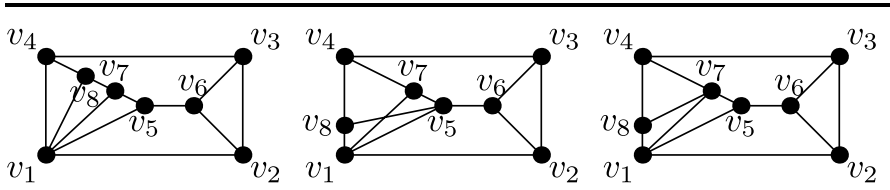

The lines $v_{1} v_{2}, v_{3} v_{4}$, and $v_{5} v_{6}$ have a common nonempty intersection
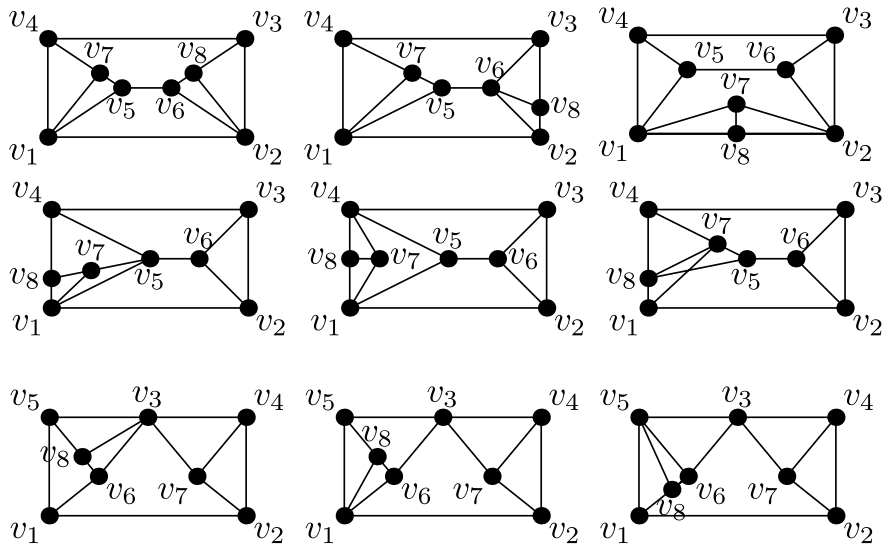

$v_{1} \nabla v_{2} \nabla v_{3}=0$

Graph (8 vert.)
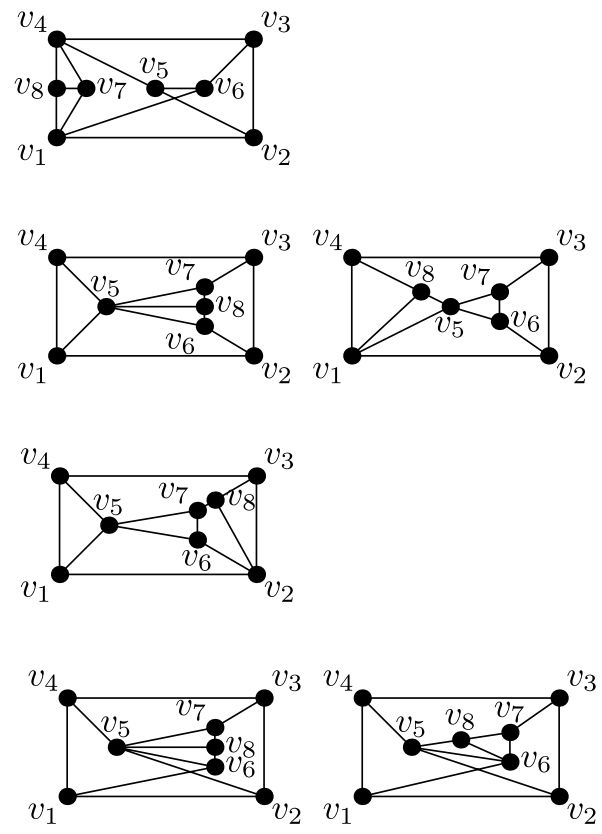

Geometric conditions

The six points $v_{1}, v_{2}, v_{3}, v_{4}, v_{5}$, and $v_{6}$ are on a conic

The lines $v_{1} v_{2}, v_{3} v_{4}$, and $v_{5} p$, where $p=\left[v_{2}, v_{6} ; v_{3}, v_{7}\right]$, have a common nonempty intersection

The lines $v_{1} v_{2}, v_{3} v_{4}$, and $v_{5} p$, where $p=\left[v_{2}, v_{6} ; v_{7}, v_{8}\right]$, have a common nonempty intersection

The six points $v_{1}, v_{2}, v_{3}, v_{4}, v_{5}$, and $p$, where $p=\left[v_{1}, v_{6} ; v_{3}, v_{7}\right]$, are on a conic 
Table 1 (Continued)

\section{Graph (8 vert.)}
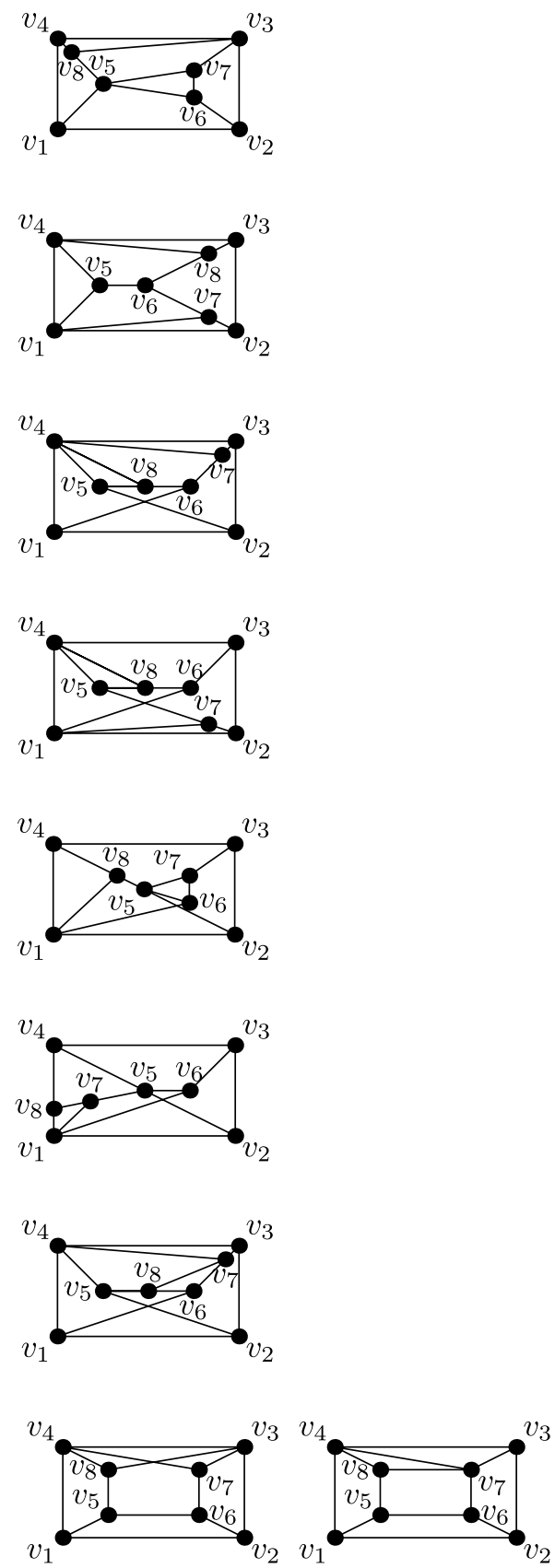

Geometric conditions

The lines $v_{1} v_{2}, v_{3} p$, and $v_{5} q$, where $p=\left[v_{1}, v_{4} ; v_{5}, v_{8}\right]$ and $q=\left[v_{2}, v_{6} ; v_{3}, v_{7}\right]$, have a common nonempty intersection

The lines $v_{5} v_{6}, v_{1} p$, and $v_{4} q$, where $p=\left[v_{2}, v_{3} ; v_{6}, v_{7}\right]$ and $q=\left[v_{2}, v_{3} ; v_{6}, v_{8}\right]$, have a common nonempty intersection

The six points $v_{1}, v_{2}, v_{4}, v_{6}, p$, and $q$, where $p=\left[v_{2}, v_{3} ; v_{6}, v_{7}\right]$ and $q=\left[v_{2}, v_{5} ; v_{6}, v_{8}\right]$, are on a conic

The six points $v_{1}, v_{3}, v_{4}, v_{6}, p$, and $q$, where $p=\left[v_{2}, v_{3} ; v_{5}, v_{7}\right]$ and $q=\left[v_{5}, v_{7} ; v_{6}, v_{8}\right]$, are on a conic

The six points $v_{1}, v_{2}, v_{3}, v_{5}, p$, and $q$, where $p=\left[v_{1}, v_{6} ; v_{3}, v_{7}\right]$ and $q=\left[v_{3}, v_{4} ; v_{5}, v_{8}\right]$, are on a conic

The six points $v_{1}, v_{2}, v_{3}, v_{5}, v_{6}$, and $q$, where $p=\left[v_{1}, q ; v_{3}, v_{4}\right]$ and $q=\left[v_{5}, v_{7} ; v_{4}, v_{8}\right]$, are on a conic

The six points $v_{1}, v_{2}, v_{4}, v_{5}, p$, and $q$, where $p=\left[v_{1}, v_{6} ; v_{5}, v_{8}\right]$ and $q=\left[p, v_{7} ; v_{2}, v_{3}\right]$, are on a conic

The three points $\left[v_{1}, v_{4} ; v_{2}, v_{3}\right]$, $\left[v_{1}, v_{5} ; v_{2}, v_{6}\right]$, and $\left[v_{5}, v_{8} ; v_{6}, v_{7}\right]$ are on one line 
Table 1 (Continued)

\begin{tabular}{|c|c|}
\hline Graph (8 vert.) & Sufficient geometric conditions \\
\hline & $\begin{array}{l}\text { The three points }\left[v_{1}, v_{2} ; v_{6}, v_{7}\right],\left[v_{1}, p ; v_{6}, v_{8}\right] \text {, and } \\
{\left[p, q ; v_{3}, v_{8}\right] \text {, where } p=\left[v_{2}, v_{4} ; v_{5}, v_{8}\right] \text { and }} \\
q=\left[v_{1}, v_{5} ; v_{3}, v_{4}\right] \text {, are on one line, AND the lines } \\
p^{\prime} v_{2}, q^{\prime} v_{3} \text {, and } v_{6} v_{7} \text { have a common nonempty } \\
\text { intersection, where } p^{\prime}=\left[r^{\prime}, s^{\prime} ; v_{1}, v_{6}\right] \text {, } \\
q^{\prime}=\left[r^{\prime}, s^{\prime} ; v_{6}, v_{8}\right], r^{\prime}=\left[v_{1}, v_{4} ; v_{2}, v_{5}\right] \text {, and } \\
s^{\prime}=\left[v_{3}, v_{4} ; v_{5}, v_{8}\right]\end{array}$ \\
\hline$v_{1}$ & $\begin{array}{l}\text { The six points } v_{1}, v_{4}, v_{7}, v_{8}, p, \text { and } q \text {, where } \\
p=\left[r, s ; v_{3}, v_{4}\right], q=\left[r, s ; v_{5}, v_{8}\right], \\
r=\left[v_{1}, v_{2} ; v_{5}, v_{6}\right] \text {, and } s=\left[v_{2}, v_{3} ; v_{6}, v_{7}\right] \text {, are on a } \\
\text { conic, AND the six points } v_{1}, v_{2}, v_{6}, v_{7}, p^{\prime} \text {, and } q^{\prime} \text {, } \\
\text { where } p^{\prime}=\left[r^{\prime}, s^{\prime} ; v_{2}, v_{3}\right], q^{\prime}=\left[r^{\prime}, s^{\prime} ; v_{5}, v_{6}\right] \text {, } \\
r^{\prime}=\left[v_{1}, v_{4} ; v_{5}, v_{8}\right] \text {, and } s^{\prime}=\left[v_{3}, v_{4} ; v_{7}, v_{8}\right] \text {, are on } \\
\text { a conic }\end{array}$ \\
\hline$v_{1}$ & $\begin{array}{l}\text { The six points } v_{1}, v_{3}, v_{4}, v_{5}, v_{7}, \text { and } p \text {, where } \\
p=\left[v_{1}, q ; v_{7}, v_{8}\right], q=\left[r, s ; v_{2}, v_{3}\right] \\
r=\left[v_{3}, v_{6} ; v_{7}, v_{8}\right] \text {, and } s=\left[v_{1}, v_{6} ; v_{2}, v_{8}\right] \text {, are on a } \\
\text { conic, AND the six points } v_{1}, v_{2}, v_{3}, v_{6}, v_{8} \text {, and } p^{\prime} \text {, } \\
\text { where } p^{\prime}=\left[v_{3}, q^{\prime} ; v_{7}, v_{8}\right], q^{\prime}=\left[r^{\prime}, s^{\prime} ; v_{1}, v_{4}\right] \text {, } \\
r^{\prime}=\left[v_{1}, v_{5} ; v_{7}, v_{8}\right] \text {, and } s^{\prime}=\left[v_{3}, v_{5} ; v_{4}, v_{7}\right] \text {, are on } \\
\text { a conic }\end{array}$ \\
\hline
\end{tabular}

Acknowledgements The authors are grateful to B. Edixhoven for rousing our interest to the subject, to W. Whiteley for fruitful discussions on geometric conditions, to A. Sossinski, V. Goryunov, and A. Perucca for useful remarks, to $\mathrm{S}$. Speed for the information on graph classification, to an unknown reviewer for excellent suggestions, and to the Mathematisch Instituut of Universiteit Leiden for hospitality and excellent working conditions. The second author is grateful to Liverpool University for the organization of a fruitful visit.

\section{References}

1. Barnabei, M., Brini, A., Rota, G.-C.: On the exterior calculus of invariant theory. J. Algebra 96(1), 120-160 (1985)

2. Berg, A.R., Jordán, T.: A proof of Connelly's conjecture on 3-connected circuits of the rigidity matroid. J. Combin. Theory Ser. B 88(1), 77-97 (2003)

3. Bochnak, J., Coste, M., Roy, M.-F.: Géométrie Algébrique Réelle. Ergebnisse der Mathematik und ihrer Grenzgebiete, Folge 3, vol. 12. Springer, Berlin (1987)

4. Bolker, E.D., Crapo, H.: Bracing rectangular frameworks, I. SIAM. J. Appl. Math. 36(3), 473-490 (1979)

5. Caspar, D.L.D., Klug, A.: Physical principles in the construction of regular viruses. In: Proceedings of Cold Spring Harbor Symposium on Quantitative Biology, vol. 27, pp. 1-24 (1962)

6. Connelly, R.: Rigidity. In: Gruber, P.M., Wills, J.M. (eds.) Handbook of Convex Geometry, vol. 44, Chap. 1.7, pp. 223-271. North-Holland, Amsterdam (1993)

7. Connelly, R., Whiteley, W.: Second-order rigidity and prestress stability for tensegrity frameworks. SIAM J. Discrete Math. 9(3), 453-491 (1996)

8. de Guzmán, M.: Finding tensegrity forms. Preprint (2004)

9. de Guzmán, M., Orden, D.: Finding tensegrity structures: geometric and symbolic approaches. In: Proceedings of EACA-2004, pp. 167-172 (2004) 
10. de Guzmán, M., Orden, D.: From graphs to tensegrity structures: geometric and symbolic approaches. Publ. Mat. 50, 279-299 (2006)

11. Doubilet, P., Rota, G.-C., Stein, J.: On the foundations of combinatorial theory. IX., Combinatorial methods in invariant theory. Stud. Appl. Math. 53, 185-216 (1974)

12. Ingber, D.E.: Cellular tensegrity: defining new rules of biological design that govern the cytoskeleton. J. Cell Sci. 104, 613-627 (1993)

13. Jackson, B., Jordán, T.: Connected rigidity matroids and unique realizations of graphs. J. Combin. Theory Ser. B 94(1), 1-29 (2005)

14. Laman, G.: On graphs and rigidity of plane skeletal structures. J. Eng. Math. 4, 331-340 (1970)

15. Maxwell, J.C.: On reciprocal figures and diagrams of forces. Philos. Mag. 4(27), 250-261 (1864)

16. Motro, R.: Tensegrity: Structural Systems for the Future. Kogan Page Science, London (2003)

17. Orden, D., Rote, G., Santos, F., Servatius, B., Servatius, H., Whiteley, W.: Non-crossing frameworks with non-crossing reciprocals. Discrete Comput. Geom. 32(4), 567-600 (2004)

18. Roth, B., Whiteley, W.: Tensegrity frameworks. Trans. Amer. Math. Soc. 265(2), 419-446 (1981)

19. Servatius, B.: Tensegrities. PAMM 7(1), 1070101-1070102 (2007)

20. Skelton, R.E.: Deployable tendon-controlled structure. United States Patent 5642590 (1 July, 1997)

21. Snelson, K.: http://www.kennethsnelson.net

22. Speed, S.: http://www.msci.memphis.edu/ speeds/

23. Sturmfels, B., Whiteley, W.: On the synthetic factorization of projectively invariant polynomials. J. Symb. Comput. 11, 439-453 (1991)

24. Tibert, A.G.: Deployable tensegrity structures for space applications. Ph.D. thesis, Royal Institute of Technology, Stockholm (2002)

25. White, N.L., Whiteley, W.: The algebraic geometry of stresses in frameworks. SIAM J. Algebr. Discrete Methods 4(4), 481-511 (1983)

26. Whiteley, W.: Rigidity and scene analysis. In: Goodman, J.E., O'Rourke, J. (eds.) Handbook of Discrete and Computational Geometry, Chap. 49, pp. 893-916. CRC, New York (1997) 